\title{
Uniformizing functions for certain Shimura curves, in the case $D=6$
}

\author{
by \\ P. Bayer and A. Travesa (Barcelona)
}

Introduction. Let $\mathbb{H}_{6}$ be the rational quaternion algebra of discriminant $D=6$, which we consider embedded into $\mathbf{M}(2, \mathbb{R})$. Let $\Gamma_{6} \subseteq \mathbf{S L}(2, \mathbb{R})$ be the group of units of norm 1 in a maximal order $\mathcal{O}_{6} \subseteq \mathbb{H}_{6}$ of discriminant $D=6$. The quotient group $\bar{\Gamma}_{6}=\Gamma_{6} /\{ \pm 1\} \subseteq \operatorname{PSL}(2, \mathbb{R})$ is a co-compact Fuchsian group whose action on the upper half-plane $\mathcal{H}$ gives rise to a nonsingular complete curve $X_{6}$ of genus zero. According to Shimura, the curve $X_{6}$ has a canonical model defined over $\mathbb{Q}$. Our purpose is to compute a complex uniformizing function $t_{6}$ of $X_{6}$ relying on the fundamental domain for $\bar{\Gamma}_{6}$ obtained in [1], and to derive from it a canonical model, $j_{6}$.

The function $j_{6}$ is an analog of the elliptic modular function $j$, which is obtained from the split quaternion algebra $\mathbf{M}(2, \mathbb{Q})$, of discriminant $D=1$. However, the functions $j$ and $j_{6}$ present notable differences. The function $j$ is automorphic under the modular group $\operatorname{PSL}(2, \mathbb{Z})$, which is a triangle Fuchsian group endowed with parabolic transformations. The function $j_{6}$ is automorphic for a quadrilateral Fuchsian group without parabolic transformations, $\bar{\Gamma}_{6} \subseteq \mathbf{P S L}(2, \mathbb{R})$. The lack of cusps in this case prevents the use of Fourier series expansions.

The complex uniformizing function we aim to determine satisfies a nonlinear differential equation of the third order, which can be obtained from the fundamental domain. In principle, it depends on eight parameters. Six of these parameters can be specified uniquely by prescribing the values of $t_{6}$ at three points, but the remaining two need to be determined by other means. In order to find their value, we simultaneously undertake the uniformization of several quotients of $X_{6}$ : the curves $X_{6}^{(2)}, X_{6}^{(3)}, X_{6}^{(6)}, X_{6}^{+}$, attached to a group $\bar{\Gamma}_{6}^{+} / \bar{\Gamma}_{6}$ of involutions of $X_{6}$.

2000 Mathematics Subject Classification: 11G18, 14G35, 30F35, 33E30.

Partially supported by MCYT BFM2003-01898 and MEC MTM2006-04895. 
The Fuchsian groups uniformizing the curves $X_{6}^{(2)}, X_{6}^{(3)}, X_{6}^{+}$are triangle groups, but the Fuchsian group uniformizing $X_{6}^{(6)}$ is again a quadrilateral group. The associated differential equations in the triangle case are determined by particular choices of the uniformizing functions. But from the two quadrilateral cases, $X_{6}$ and $X_{6}^{(6)}$, four accessory parameters remain. Two of them will be determined from algebraic equations of the coverings, which we compute beforehand. Formal integration of the differential equations will provide the other two.

Once the five uniformizing equations are fully determined, we integrate them to obtain the uniformizing functions $t_{6}, t_{6}^{(2)}, t_{6}^{(3)}, t_{6}^{(6)}, t_{6}^{+}$. We expand these functions as power series in local uniformizing parameters $q_{P}$ attached to the vertices $P$ of fundamental half domains. Moreover, we make explicit the local development of $t_{6}$ at the special complex multiplication points of $X_{6}$. Knowledge of the functions $q_{P}$ involves the local isotropy at $P$, the exact determination of the integration constants, and the right choice of local normalizing factors.

In the last part of the paper, we use the values of our functions at the elliptic points and the special complex multiplication points to derive the canonical models of all the curves involved.

We are indebted to the anonymous referee, whose valuable comments have enabled us to improve the final version of the manuscript.

1. The Fuchsian group $\bar{\Gamma}_{6}$ and the Shimura curve $X_{6}$. Let $\mathbb{H}_{6}$ be the rational quaternion algebra with basis $\{1, \mathrm{I}, \mathrm{J}, \mathrm{K}\}$ and defined by $\mathrm{I}^{2}=3$, $\mathrm{J}^{2}=-1$, IJ $=-\mathrm{JI}=\mathrm{K}$. Its discriminant $D$, given by the product of the places $v$ where $\mathbb{H}_{6} \otimes \mathbb{Q}_{v}$ is a division algebra, is equal to 6 . The algebra $\mathbb{H}_{6}$ is the rational non-split $(D \neq 1)$ quaternion algebra of lowest discriminant which is unramified at $\infty$. In particular, it can be embedded into $\mathbf{M}(2, \mathbb{R})$.

Throughout the paper we fix the embedding $\Phi: \mathbb{H}_{6} \rightarrow \mathbf{M}(2, \mathbb{R})$ given by

$$
x+y \mathrm{I}+z \mathrm{~J}+t \mathrm{~K} \mapsto\left[\begin{array}{cc}
x+y \sqrt{3} & z+t \sqrt{3} \\
-(z-t \sqrt{3}) & x-y \sqrt{3}
\end{array}\right] .
$$

The reduced trace and the reduced norm of a quaternion are given by $\operatorname{tr}(x+$ $y \mathrm{I}+z \mathrm{~J}+t \mathrm{~K})=2 x$ and $\mathrm{n}(x+y \mathrm{I}+z \mathrm{~J}+t \mathrm{~K})=x^{2}-3 y^{2}+z^{2}-3 t^{2}$. They agree with the trace and the determinant of the matrix $\Phi(x+y \mathrm{I}+z \mathrm{~J}+t \mathrm{~K})$.

All maximal orders in $\mathbb{H}_{6}$ are conjugate, and we fix the representative in this conjugacy class to be $\mathcal{O}_{6}:=\mathbb{Z}[1, \mathrm{I}, \mathrm{J},(1+\mathrm{I}+\mathrm{J}+\mathrm{K}) / 2]$. Let $\left(\mathcal{O}_{6}\right)_{1}^{*}=$ $\left\{\gamma \in \mathcal{O}_{6}: \mathrm{n}(\gamma)=1\right\}$ be the group of its units of reduced norm equal to one. The group $\left(\mathcal{O}_{6}\right)_{1}^{*}$ can be identified with its image $\Gamma_{6} \subseteq \mathbf{S L}(2, \mathbb{R})$ under $\Phi$. This group admits the following description, given in [1]: 


$$
\Gamma_{6}=\left\{\gamma=\frac{1}{2}\left[\begin{array}{cc}
\alpha & \beta \\
-\beta^{\prime} & \alpha^{\prime}
\end{array}\right]: \alpha, \beta \in \mathbb{Z}[\sqrt{3}], \operatorname{det} \gamma=1, \alpha \equiv \beta \equiv \alpha \sqrt{3}(\bmod 2)\right\}
$$

Here $\alpha^{\prime}$ denotes the non-trivial Galois conjugate of an element $\alpha \in \mathbb{Q}(\sqrt{3})$. The projection $\bar{\Gamma}_{6}:=\Gamma_{6} /\{ \pm 1\}$ in $\operatorname{PSL}(2, \mathbb{R})$ is a Fuchsian group which does not have parabolic transformations.

Let $\mathcal{H}=\{z \in \mathbb{C}: \operatorname{Im} z>0\}$ be the complex upper half-plane. The group $\operatorname{PSL}(2, \mathbb{R})=\mathbf{G L}^{+}(2, \mathbb{R}) / \mathbb{R}^{*}$ acts freely on $\mathcal{H}$ by homographic transformations $\gamma(z)=(a z+b) /(c z+d)$, defined by matrices $\gamma=\left[\begin{array}{ll}a & b \\ c & d\end{array}\right] \in \mathbf{G L}^{+}(2, \mathbb{R})$.

The action of $\bar{\Gamma}_{6}$ on $\mathcal{H}$ yields a compact Riemann surface $\bar{\Gamma}_{6} \backslash \mathcal{H}$ or, equivalently, a projective non-singular curve $X_{6}$, which is of genus zero. Note that the explicit identification of $\bar{\Gamma}_{6} \backslash \mathcal{H}$ with $\mathbf{P}^{1}(\mathbb{C})$ is by no means obvious, since it implies the construction of coordinate functions automorphic under $\bar{\Gamma}_{6}$. By a theorem due to Shimura, we know that the curve $X_{6}$ has a model defined over $\mathbb{Q}$ without rational points.

A fundamental domain for the action of $\bar{\Gamma}_{6}$ in $\mathcal{H}$, together with some distinguished complex multiplication points in it, called special complex multiplication points (SCM), was computed in [1]. We summarize some of its properties.

TheOREM 1.1. Consider the hyperbolic hexagon $\left[P_{1}, P_{2}, \ldots, P_{6}\right]$ with vertices $P_{i}$ given in Table 1 . For each $i$, the isotropy group at $P_{i}$ under $\bar{\Gamma}_{6}$ is generated by the class of the matrix $\eta_{i}$ in Table 2 . Under the identifications
(a) $\eta_{2}\left[P_{3}, P_{2}\right]=\left[P_{1}, P_{2}\right]$,
(b) $\eta_{4}\left[P_{3}, P_{4}\right]=\left[P_{5}, P_{4}\right]$,
(c) $\eta_{6}\left[P_{5}, P_{6}\right]=\left[P_{1}, P_{6}\right]$,

the hexagon yields a fundamental domain for the action of $\bar{\Gamma}_{6}$ in $\mathcal{H}$ (see Figure 1$)$. The vertices $P_{1} \equiv P_{3} \equiv P_{5}\left(\bmod \bar{\Gamma}_{6}\right)$ and $P_{6}$ are elliptic of order 2 ; the remaining vertices $P_{2}, P_{4}$ are elliptic of order 3 . The points $P_{0}$, $P_{7} \equiv P_{8}\left(\bmod \bar{\Gamma}_{6}\right)$ in Table 1 are a full set of representatives of the special complex multiplication (SCM) points of the genus zero curve $X_{6}$.

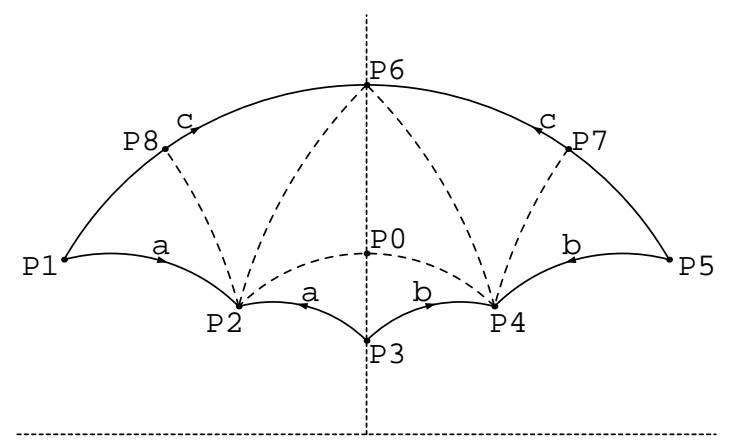

Fig. 1. Fundamental domain for $X_{6}$ 
Table 1. Vertices of a fundamental domain for $X_{6}$ and SCM points

\begin{tabular}{cc|cc|cc}
\hline$P_{1}$ & $(-\sqrt{3}+i) / 2$ & $P_{2}$ & $(-1+i) /(1+\sqrt{3})$ & $P_{3}$ & $(2-\sqrt{3}) i$ \\
$P_{4}$ & $(1+i) /(1+\sqrt{3})$ & $P_{5}$ & $(\sqrt{3}+i) / 2$ & $P_{6}$ & $i$ \\
$P_{7}$ & $(1+\sqrt{2} i) / \sqrt{3}$ & $P_{8}$ & $(-1+\sqrt{2} i) / \sqrt{3}$ & $P_{0}$ & $(\sqrt{6}-\sqrt{2}) i / 2$ \\
\hline
\end{tabular}

Table 2. Matrices representing generators for the isotropy groups at the vertices of the hexagon

\begin{tabular}{|c|c|c|c|c|c|c|c|c|}
\hline$\eta_{1}$ & & {$\left[\begin{array}{l}\sqrt{3} \\
-2\end{array}\right.$} & $\left.\begin{array}{c}2 \\
-\sqrt{3}\end{array}\right]$ & $\eta_{2} \quad \frac{1}{2}$ & {$\left[\begin{array}{cc}1+\sqrt{3} & 3-\sqrt{3} \\
-3-\sqrt{3} & 1-\sqrt{3}\end{array}\right]$} & $\eta_{3}$ & $\begin{array}{c}0 \\
2+\sqrt{3}\end{array}$ & $\left.\begin{array}{c}-2+\sqrt{3} \\
0\end{array}\right]$ \\
\hline$\eta_{4}$ & $\frac{1}{2}$ & $\begin{array}{l}1+\sqrt{3} \\
3+\sqrt{3}\end{array}$ & $\left.\begin{array}{c}-3+\sqrt{3} \\
1-\sqrt{3}\end{array}\right]$ & $\eta_{5}$ & {$\left[\begin{array}{cc}\sqrt{3} & -2 \\
2 & -\sqrt{3}\end{array}\right]$} & $\eta_{6}$ & {$\left[\begin{array}{c}0 \\
-1\end{array}\right.$} & $\left.\begin{array}{l}1 \\
0\end{array}\right]$ \\
\hline
\end{tabular}

2. The Fuchsian group $\bar{\Gamma}_{6}^{+}$and quotients of $X_{6}$. Let $N\left(\mathcal{O}_{6}\right)$ be the normalizer of $\mathcal{O}_{6}$ in $\mathbb{H}_{6}$. The elements of $N\left(\mathcal{O}_{6}\right)$ of positive reduced norm define a subgroup whose image in $\mathbf{G L}^{+}(2, \mathbb{R})$ will be denoted by $\Gamma_{6}^{+}$. The group $\Gamma_{6}$ is contained in $\Gamma_{6}^{+}$as a normal subgroup and the quotient $\bar{\Gamma}_{6}^{+} / \bar{\Gamma}_{6}$ is isomorphic to $(\mathbb{Z} / 2 \mathbb{Z})^{2}$. Its classes are represented by elements $w_{d} \in \mathcal{O}_{6}$ of norm $d$ dividing $D=6$. They give rise to involutions of the curve $X_{6}$, denoted $\omega_{d}$ (cf. [8]). We shall consider, together with the Shimura curve $X_{6}$, its quotients $X_{6}^{(d)}:=X_{6} /\left\langle\omega_{d}\right\rangle$ and $X_{6}^{+}:=X_{6} /\left\langle\left\{\omega_{d}: d \mid 6\right\}\right\rangle$. All these Shimura curves fit in the following diagram of Galois covers of degree two:

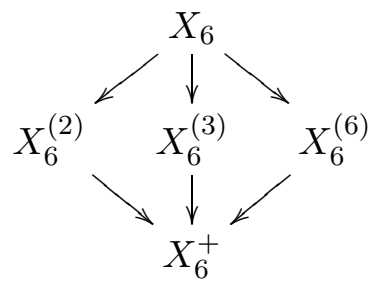

In what follows, we shall compute fundamental domains for the curves so defined.

First, consider the elements $w_{2}:=1+\mathrm{J}, w_{3}:=(-3-\mathrm{I}-3 \mathrm{~J}+\mathrm{K}) / 2$, $w_{3}^{\prime}:=(3+\mathrm{I}-3 \mathrm{~J}+\mathrm{K}) / 2$, and $w_{6}:=w_{2} w_{3}=-3 \mathrm{~J}+\mathrm{K}$ in $\mathcal{O}_{6}$. Their classes in $\bar{\Gamma}_{6}^{+} / \bar{\Gamma}_{6}$ define involutions $\omega_{d}, \omega_{d}^{\prime}$ of $X_{6}$. Observe that $\omega_{3}^{\prime}=\omega_{3}$, because $w_{3}^{\prime}=\eta_{6} w_{3}$ and $\eta_{6} \in \Gamma_{6}$; and $\omega_{6} \eta_{2}^{-1}=\omega_{6}$, because $\eta_{2} \in \Gamma_{6}$. Then we have the following polygon identifications: $\omega_{2}\left[P_{1}, P_{2}, P_{6}\right]=\left[P_{3}, P_{4}, P_{6}\right]$, $\omega_{2}\left[P_{2}, P_{3}, P_{6}\right]=\left[P_{4}, P_{5}, P_{6}\right], \omega_{3}\left[P_{2}, P_{3}, P_{6}\right]=\left[P_{2}, P_{6}, P_{1}\right], \omega_{3}^{\prime}\left[P_{3}, P_{4}, P_{6}\right]=$ $\left[P_{6}, P_{4}, P_{5}\right], \omega_{6}\left[P_{2}, P_{3}, P_{6}\right]=\left[P_{4}, P_{6}, P_{3}\right]$, and $\omega_{6} \eta_{2}^{-1}\left[P_{1}, P_{2}, P_{6}\right]=\left[P_{6}, P_{4}, P_{5}\right]$. 
Proposition 2.1. Figures 2 and 3 show fundamental domains for the genus zero curves $X_{6}^{(2)}, X_{6}^{(3)}$, and $X_{6}^{(6)}$. The identifications of the sides are given as follows:

\begin{tabular}{|c|c|c|c|}
\hline & $X_{6}^{(2)}$ & $X_{6}^{(3)}$ & $X_{6}^{(6)}$ \\
\hline (a) & $\eta_{4}^{-1} \omega_{2}\left[P_{2}, P_{3}\right]=\left[P_{4}, P_{3}\right]$ & $\omega_{3}\left[P_{2}, P_{3}\right]=\left[P_{2}, P_{6}\right]$ & $\omega_{6}\left[P_{0}, P_{3}\right]=\left[P_{0}, P_{6}\right]$ \\
\hline (b) & $\omega_{2}\left[P_{2}, P_{6}\right]=\left[P_{4}, P_{6}\right]$ & $\omega_{3}^{\prime}\left[P_{4}, P_{3}\right]=\left[P_{4}, P_{6}\right]$ & $\eta_{4} \omega_{6} \eta_{6}\left[P_{6}, P_{7}\right]=\left[P_{5}, P_{7}\right]$ \\
\hline (c) & $*$ & $*$ & $\eta_{4}\left[P_{3}, P_{4}\right]=\left[P_{5}, P_{4}\right]$ \\
\hline
\end{tabular}
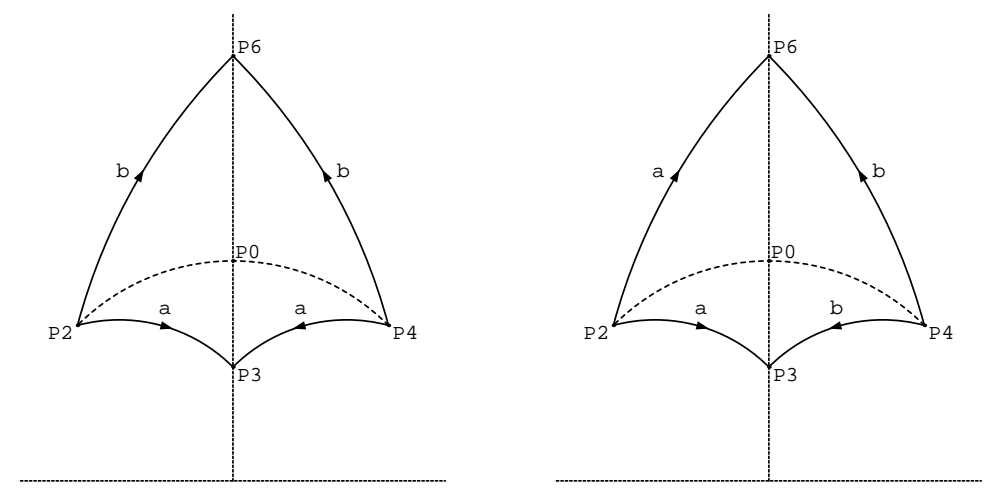

Fig. 2. Fundamental domains for $\Gamma_{6}^{(2)}$ and $\Gamma_{6}^{(3)}$
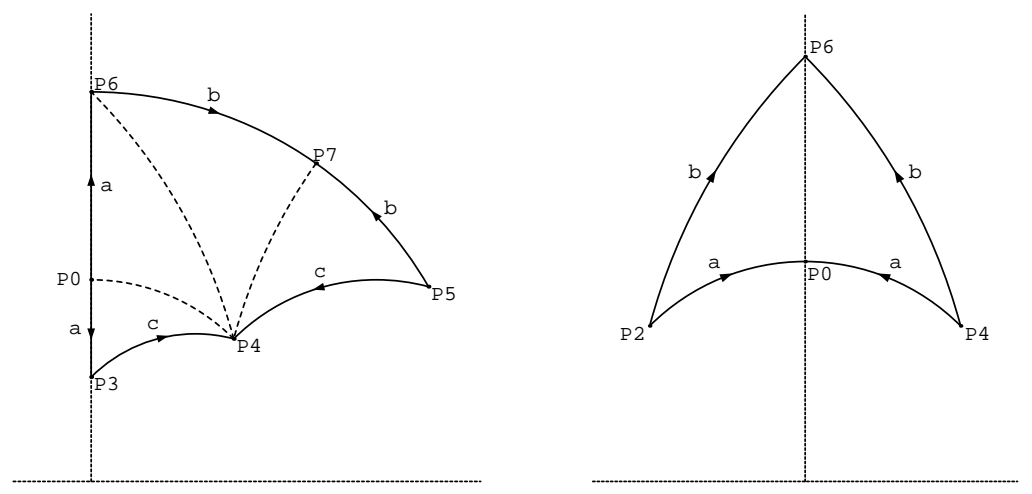

Fig. 3. Fundamental domains for $\Gamma_{6}^{(6)}$ and $\Gamma_{6}^{+}$

Now, since the curve $X_{6}^{+}$can be obtained as a quotient of any of the curves $X_{6}^{(d)}, d \neq 1$, by any involution $\omega_{d^{\prime}}, d^{\prime} \neq d, 1$, we may use the identification $\omega_{6}\left[P_{2}, P_{3}, P_{4}\right]=\left[P_{4}, P_{6}, P_{2}\right]$ to obtain a fundamental domain for the 
curve $X_{6}^{+}$from the fundamental domain for $X_{6}^{(2)}$ or, alternatively, for $X_{6}^{(3)}$. We state the result in the next proposition.

Proposition 2.2. Figure 3 shows a fundamental domain for the genus zero curve $X_{6}^{+}$. The identifications of the sides are as follows:
(a) $\omega_{6}\left[P_{2}, P_{0}\right]=\left[P_{4}, P_{0}\right]$
(b) $\omega_{6} \omega_{3}^{-1}\left[P_{2}, P_{6}\right]=\left[P_{4}, P_{6}\right]$.

3. Fundamental half domains. The fundamental domains we have obtained up to now show particular symmetries. We will take advantage of this fact to compute the uniformizing functions we are looking for, that is, functions $t$ such that $\mathbb{C}(X(\bar{\Gamma})) \simeq \mathbb{C}(t)$. For example, in Figure 1 , besides the obvious reflection in the imaginary axis, the two closed hyperbolic segments joining $P_{2}$ with $P_{6}$ and $P_{6}$ with $P_{4}$ are a unique symmetry axis for $X_{6}$, denoted $\left(P_{2}, P_{6}, P_{4}\right)$, the symmetry being the involution $\omega_{2}$ composed with the reflection in the imaginary axis. Similarly, $\left(P_{8}, P_{2}, P_{4}, P_{7}\right)$ is a symmetry axis for $X_{6}$, the symmetry being the involution $\omega_{6}$ composed with the reflection in the imaginary axis (observe that $\omega_{6}=\eta_{4} \omega_{6}=\eta_{2} \omega_{6}$ ).

But not all the symmetries above are suitable for our purposes. In fact, we require them to cut out half domains containing all the vertices of a fundamental domain exactly once. These half domains will be called fundamental. Thus the reflection in the imaginary axis is not suitable, because it cuts out half domains containing twice the vertex $P_{1} \equiv P_{3} \equiv P_{5}$ $\left(\bmod \bar{\Gamma}_{6}\right)$, while one of the vertices $P_{2}$ or $P_{4}$ is missing. Similarly, the symmetry with axis $\left(P_{8}, P_{2}, P_{0}, P_{4}, P_{7}\right)$ is not suitable; for example, it identifies the vertices $P_{1} \equiv P_{3} \equiv P_{5}$ with $P_{6}$ but $P_{7} \equiv P_{8}$ appears twice in each half domain. A convenient symmetry is the one with axis $\left(P_{2}, P_{6}, P_{4}\right)$, since it cuts out the quadrilateral $\left[P_{2}, P_{3}, P_{4}, P_{6}\right]$ as a fundamental half domain for $X_{6}$.

Proposition 3.1. Table 3 lists axes of symmetries and polygons defining fundamental half domains for the curves $X_{6}, X_{6}^{(2)}, X_{6}^{(3)}, X_{6}^{(6)}$, and $X_{6}^{+}$. The fundamental half domains are cut out of the fundamental domains in 1.1, 2.1 and 2.2 .

Table 3. Fundamental half domains

\begin{tabular}{ccc}
\hline Curve & Axis of symmetry & Polygons \\
\hline$X_{6}$ & $\left(P_{2}, P_{6}, P_{4}\right)$ & {$\left[P_{2}, P_{3}, P_{4}, P_{6}\right]$} \\
$X_{6}^{(2)}$ & $\left(P_{3}, P_{6}\right)$ & {$\left[P_{3}, P_{4}, P_{6}\right]$} \\
$X_{6}^{(3)}$ & $\left(P_{2}, P_{4}\right)$ & {$\left[P_{2}, P_{4}, P_{6}\right]$} \\
$X_{6}^{(6)}$ & $\left(P_{0}, P_{4}, P_{7}\right)$ & {$\left[P_{0}, P_{4}, P_{7}, P_{6}\right]$} \\
$X_{6}^{+}$ & $\left(P_{0}, P_{6}\right)$ & {$\left[P_{0}, P_{4}, P_{6}\right]$} \\
\hline
\end{tabular}


At this point, we compute the angles at the different vertices of the polygons defining the corresponding fundamental half domains. Since the angle at any vertex in a fundamental half domain equals half the sum of the angles at the homologous vertices of the complete fundamental domain, we obtain the results displayed in Table 4.

Table 4. Angles at the vertices of fundamental half domains

\begin{tabular}{ccccccc}
\hline & $P_{0}$ & $P_{2}$ & $P_{3}$ & $P_{4}$ & $P_{6}$ & $P_{7}$ \\
\hline$X_{6}$ & $*$ & $\pi / 3$ & $\pi / 2$ & $\pi / 3$ & $\pi / 2$ & $*$ \\
$X_{6}^{(2)}$ & $*$ & $*$ & $\pi / 4$ & $\pi / 3$ & $\pi / 4$ & $*$ \\
$X_{6}^{(3)}$ & $\pi$ & $\pi / 6$ & $*$ & $\pi / 6$ & $\pi / 2$ & $*$ \\
$X_{6}^{(6)}$ & $\pi / 2$ & $*$ & $*$ & $\pi / 3$ & $\pi / 2$ & $\pi / 2$ \\
$X_{6}^{+}$ & $\pi / 2$ & $*$ & $*$ & $\pi / 6$ & $\pi / 4$ & $*$ \\
\hline
\end{tabular}

4. Equations for the covers. The next step in our computation is to determine the equations of the covers. We shall begin by controlling their ramification. The ramification points can be easily seen from the pictures of the fundamental domains, and we have the following result.

Proposition 4.1. Table 6 shows the ramification points of all the covers of degree two that we have. The different ways in which they are identified depend on the curve, and the identifications are shown in Table 5.

Table 5. Identification of points

\begin{tabular}{ccccccc}
\hline$X_{6}$ & $P_{0}$ & $P_{1} \equiv P_{3} \equiv P_{5}$ & $P_{2}$ & $P_{4}$ & $P_{6}$ & $P_{7} \equiv P_{8}$ \\
$X_{6}^{(2)}$ & $P_{0} \equiv P_{7}$ & $P_{3}$ & $P_{2} \equiv P_{4}$ & $P_{2} \equiv P_{4}$ & $P_{6}$ & $P_{0} \equiv P_{7}$ \\
$X_{6}^{(3)}$ & $P_{0} \equiv P_{7}$ & $P_{3} \equiv P_{6}$ & $P_{2}$ & $P_{4}$ & $P_{3} \equiv P_{6}$ & $P_{0} \equiv P_{7}$ \\
$X_{6}^{(6)}$ & $P_{0}$ & $P_{3} \equiv P_{6}$ & $P_{2} \equiv P_{4}$ & $P_{2} \equiv P_{4}$ & $P_{3} \equiv P_{6}$ & $P_{7}$ \\
$X_{6}^{+}$ & $P_{0} \equiv P_{7}$ & $P_{3} \equiv P_{6}$ & $P_{2} \equiv P_{4}$ & $P_{2} \equiv P_{4}$ & $P_{3} \equiv P_{6}$ & $P_{0} \equiv P_{7}$ \\
\hline
\end{tabular}

Table 6. All ramification and some splitting points of the covers

\begin{tabular}{ccccccc}
\hline Cover & $P_{0}$ & $P_{2}$ & $P_{3}$ & $P_{4}$ & $P_{6}$ & $P_{7}$ \\
\hline$X_{6} \rightarrow X_{6}^{(2)}$ & $P_{0} P_{7}$ & $*$ & $P_{3}^{2}$ & $P_{2} P_{4}$ & $P_{6}^{2}$ & $*$ \\
$X_{6} \rightarrow X_{6}^{(3)}$ & $P_{0} P_{7}$ & $P_{2}^{2}$ & $*$ & $P_{4}^{2}$ & $P_{3} P_{6}$ & $*$ \\
$X_{6} \rightarrow X_{6}^{(6)}$ & $P_{0}^{2}$ & $*$ & $*$ & $P_{2} P_{4}$ & $P_{3} P_{6}$ & $P_{7}^{2}$ \\
$X_{6}^{(2)} \rightarrow X_{6}^{+}$ & $P_{0}^{2}$ & $*$ & $*$ & $P_{4}^{2}$ & $P_{3} P_{6}$ & $*$ \\
$X_{6}^{(3)} \rightarrow X_{6}^{+}$ & $P_{0}^{2}$ & $*$ & $*$ & $P_{2} P_{4}$ & $P_{6}^{2}$ & $*$ \\
$X_{6}^{(6)} \rightarrow X_{6}^{+}$ & $P_{0} P_{7}$ & $*$ & $*$ & $P_{4}^{2}$ & $P_{6}^{2}$ & $*$ \\
\hline
\end{tabular}


Since the curves we are dealing with are all of genus zero, their function field can be generated by a single automorphic function in each case. Due to the fact that the group of automorphisms of $\mathbf{P}^{1}(\mathbb{C})$ is isomorphic to $\operatorname{PSL}(2, \mathbb{C})$, we may isolate a generator by requiring that it assumes three given different values at three given different points.

Proposition 4.2. For each curve $X_{6}, X_{6}^{(2)}, X_{6}^{(3)}, X_{6}^{(6)}, X_{6}^{+}$, there exists a uniformizing function, uniquely determined by three values at three vertices of the fundamental half domains in Table 3.

Proof. Since our curves are of genus zero and the Fuchsian groups attached to them admit fundamental half domains, the Riemann mapping theorem and the Schwarz reflection principle provide the existence of uniformizing functions (cf. [9]). Since $\mathbf{P S L}(2, \mathbb{C})$ is the automorphism group of $\mathbf{P}^{1}(\mathbb{C})$, the uniformizing functions are determined by their values at three points.

Table 7. Values of the uniformizing functions at the vertices

\begin{tabular}{ccccccc}
\hline$t$ & $P_{0}$ & $P_{2}$ & $P_{3}$ & $P_{4}$ & $P_{6}$ & $P_{7}$ \\
\hline$t_{6}$ & $*$ & $a$ & 0 & 1 & $\infty$ & $*$ \\
$t_{6}^{(2)}$ & $*$ & $*$ & 0 & 1 & $\infty$ & $*$ \\
$t_{6}^{(3)}$ & $*$ & 0 & $*$ & 1 & $\infty$ & $*$ \\
$t_{6}^{(6)}$ & 0 & $*$ & $*$ & 1 & $\infty$ & $b$ \\
$t_{6}^{+}$ & 0 & $*$ & $*$ & 1 & $\infty$ & $*$ \\
\hline
\end{tabular}

We choose or name some initial values for the functions we are aiming at in accordance with Table 7 . Then we have the following theorem.

TheOREM 4.3. The conditions in Table 7 define uniformizing functions $t_{6}, t_{6}^{(2)}, t_{6}^{(3)}, t_{6}^{(6)}, t_{6}^{+}$which fulfil the following algebraic relations:
(a) $4 t_{6}^{+} t_{6}^{(2)}=\left(t_{6}^{(2)}+1\right)^{2}$,
(c) $4 t_{6}^{(2)}\left(2 t_{6}^{(3)}-1\right)^{2}=\left(t_{6}^{(2)}+1\right)^{2}$,
(b) $t_{6}^{+}=\left(2 t_{6}^{(3)}-1\right)^{2}$,
(e) $4 t_{6} t_{6}^{(3)}=\left(t_{6}+1\right)^{2}$
(d) $t_{6}^{2}=t_{6}^{(2)}$,
(g) $2 t_{6} t_{6}^{(6)}=i\left(t_{6}-i\right)^{2}$,
(f) $t_{6}^{+}+t_{6}^{(6)}\left(t_{6}^{(6)}-2\right)=0$,
(i) $\left(t_{6}^{(2)}+1\right)^{2}+4 t_{6}^{(2)} t_{6}^{(6)}\left(t_{6}^{(6)}-2\right)=0$,
(h) $4 t_{6}^{2} t_{6}^{+}=\left(t_{6}^{2}+1\right)^{2}$,
(j) $\left(2 t_{6}^{(3)}-1\right)^{2}+t_{6}^{(6)}\left(t_{6}^{(6)}-2\right)=0$.

All these functions take real values on the boundaries of their respective half domains. Moreover, we have the following particular values:
(k) $t_{6}^{(2)}\left(P_{0}\right)=-1$,
(l) $t_{6}^{(3)}\left(P_{0}\right)=1 / 2$,
(m) $b=t_{6}^{(6)}\left(P_{7}\right)=2$,
(n) $a=t_{6}\left(P_{2}\right)=-1$,
(o) $t_{6}\left(P_{0}\right)=i$
(p) $t_{6}\left(P_{7}\right)=-i$. 
Proof. We write for the moment $c:=t_{6}^{(2)}\left(P_{0}\right)$ and compute some divisors of the functions, taking into account the ramification given in Proposition 4.1:

$$
\operatorname{div}\left(t_{6}^{(2)}-c\right)\left(1-\frac{c}{t_{6}^{(2)}}\right)=\operatorname{div}\left(t_{6}^{+}\right), \quad \operatorname{div}\left(t_{6}^{(2)}-1\right)\left(1-\frac{1}{t_{6}^{(2)}}\right)=\operatorname{div}\left(t_{6}^{+}-1\right)
$$

Since for genus 0 curves each zero degree divisor determines a function up to a non-zero constant factor, there exist non-zero constants $A, B \in \mathbb{C}$ such that

$$
A\left(t_{6}^{(2)}-c\right)\left(1-\frac{c}{t_{6}^{(2)}}\right)=t_{6}^{+}, \quad B\left(t_{6}^{(2)}-1\right)\left(1-\frac{1}{t_{6}^{(2)}}\right)=t_{6}^{+}-1 .
$$

Solving the system, we obtain (a) and the value $c=-1$.

Similarly, considering $d:=t_{6}^{(3)}\left(P_{0}\right)$ and computing $\operatorname{div}\left(t_{6}^{(3)}-d\right)^{2}=$ $\operatorname{div}\left(t_{6}^{+}\right), \operatorname{div}\left(t_{6}^{(3)}-1\right) t_{6}^{(3)}=\operatorname{div}\left(t_{6}^{+}-1\right)$, we obtain $(\mathrm{b})$ and the value $d=1 / 2$.

(c) is an immediate consequence of (a) and (b). The remaining algebraic relations are obtained analogously.

The defining conditions of the automorphic triangle functions $t_{6}^{(2)}, t_{6}^{(3)}, t_{6}^{+}$ uniquely determine the conformal representations of the half domains in Table 3 in $\mathcal{H}$. Thus, these functions are real on the boundary of their half domains. The algebraic relations satisfied by the quadrangular functions $t_{6}^{(6)}$ and $t_{6}$ imply that these functions are real on the boundary of their respective half domains. In order to decide which of the two values $i$ or $-i$ equals $t_{6}\left(P_{0}\right)$, we note that, since the function $t_{6}$ respects the orientation, the interior of the quadrilateral $\left[P_{2}, P_{3}, P_{4}, P_{6}\right]$ has to be mapped into $\mathcal{H}$.

5. Uniformizing differential equations. We review the necessary definitions and facts regarding Schwarzian derivatives, drawn mostly from $[2],[3]$ and [5].

Definition 5.1. Let $f(z)$ be a non-constant smooth function and let $D(f, z)$ be the usual derivative. The Schwarzian derivative of $f$ is defined as

$$
D s(f, z)=\frac{2 D(f, z) D^{3}(f, z)-3 D^{2}(f, z)^{2}}{D(f, z)^{2}} ;
$$

and the automorphic derivative of $f$ is defined as

$$
D a(f, z)=\frac{D s(f, z)}{D(f, z)^{2}} .
$$

Neither the Schwarzian derivative nor the automorphic derivative are derivations in the usual sense, but these differential operators have some properties similar to those of the standard derivation. We now state some of these properties. 


\section{Proposition 5.2.}

(a) Let $f(z), g(z)$ be non-constant smooth functions whose composition $g \circ f$ is defined. Then the automorphic derivative satisfies the following chain rule:

$$
D a(g \circ f, z)=D a(g, f(z))+\frac{D a(f, z)}{D(g, f(z))^{2}} .
$$

(b) Suppose that $f(z)=w$ is a smooth function whose inverse function is $\mathbf{P G L}(2, \mathbb{C})$-multivalued. Then the Schwarzian derivative $D s\left(f^{-1}, w\right)$ is single valued and $D s\left(f^{-1}, w\right)=-D a(f, z)$, where $f^{-1}(w)=z$.

(c) For a homographic transformation $\gamma(z)=(a z+b) /(c z+d), \gamma=$ $\left[\begin{array}{ll}a & b \\ c & d\end{array}\right] \in \mathbf{G L}(2, \mathbb{C})$, we have $\operatorname{Da}(\gamma, z)=0$. In particular, Da $(f \circ \gamma, z)$ $=\operatorname{Da}(f, \gamma(z))$ for any function $f(z)$.

(d) Conversely, $D a(\gamma, z)=0$ implies that $\gamma(z)=(a z+b) /(c z+d)$ for some $\gamma=\left[\begin{array}{ll}a & b \\ c & d\end{array}\right] \in \mathbf{M}(2, \mathbb{C})$.

For a Fuchsian group $\bar{\Gamma} \subseteq \mathbf{P S L}(2, \mathbb{R})$, and a $\bar{\Gamma}$-automorphic function $f$, we deduce the $\bar{\Gamma}$-invariance of $D a(f, z)$.

Corollary 5.3. The automorphic derivative $D a(f, z)$ of a $\bar{\Gamma}$-automorphic function, $f(z)$, is again a $\bar{\Gamma}$-automorphic function. That is to say, the following equality holds:

$$
D a(f, \gamma(z))=D a(f, z) \quad \text { for any } \gamma \in \bar{\Gamma} .
$$

The main tool in our approach to the differential treatment of the uniformizing functions will be the following theorem.

THEOREM 5.4. Let $\bar{\Gamma}$ be a Fuchsian group of the first kind such that the associated curve $X(\bar{\Gamma})$ is of genus 0 . Assume that we know a fundamental half domain for the action of $\bar{\Gamma}$ in $\mathcal{H}$. Suppose that $t$ is a generator of the field of $\bar{\Gamma}$-automorphic functions such that its values at the vertices of the fundamental half domain belong to $\mathbf{P}^{1}(\mathbb{R})$. Then there exists a rational function $R(t)$ such that $D a(t, z)+R(t)=0$. If $\alpha_{i} \pi$ are the internal angles at the vertices of the fundamental half domain, then

$$
R(t)=\sum \frac{1-\alpha_{i}^{2}}{\left(t-a_{i}\right)^{2}}+\sum \frac{B_{i}}{t-a_{i}},
$$

where the $B_{i}$ are constants and the summation extends over all vertices of the fundamental half domain where the function $t$ takes finite values $a_{i}$. Moreover, if the values of $t$ at all vertices are finite, then

(a) $\sum B_{i}=0$

(b) $\sum a_{i} B_{i}+\sum\left(1-\alpha_{i}^{2}\right)=0$,

(c) $\sum a_{i}^{2} B_{i}+\sum a_{i}\left(1-\alpha_{i}^{2}\right)=0$. 
But if $\infty$ is the value of $t$ at a vertex with internal angle $\alpha \pi$, then

(a) $\sum B_{i}=0$,

(b) $\sum a_{i} B_{i}+\sum\left(1-\alpha_{i}^{2}\right)-\left(1-\alpha^{2}\right)=0$.

In general, the above relations between the constants $B_{i}$, the angles $\alpha_{i}$, and the values $a_{i}$ do not suffice to determine all the constants. In our case, the relations between all our functions given in Theorem 4.3 fully determine the constants $B_{i}$ only for three of the five functions $R(t)$, namely for those associated to $t_{6}^{(2)}, t_{6}^{(3)}$, and $t_{6}^{+}$. To determine the constants that remain, we formally integrate the differential equation computing some coefficients of the expansion in a neighbourhood of the point $P_{6}$ and compare the solutions using the equations for the covers we have obtained in Theorem 4.3. When comparing solutions for $t_{6}$ and $t_{6}^{(2)}$, we obtain the value of the constant term in the differential equation for $t_{6}$; and when comparing solutions for $t_{6}^{(6)}$ and $t_{6}^{+}$, we obtain the value of the constant term in the differential equation for $t_{6}^{(6)}$. This gives the values of the automorphic derivatives.

TheOREM 5.5. The functions defined by Table 7 satisfy the differential equations $D a(t, z)+R(t)=0$, where the rational functions $R(t)$ are listed in Table 8.

Table 8. Automorphic derivatives of the functions

\begin{tabular}{cccc}
\hline Curve & $\begin{array}{c}\text { Function } \\
\text { t }\end{array}$ & Vertices & $-D a(t, z)$ \\
\hline$X_{6}$ & $t_{6}$ & {$\left[P_{2}, P_{3}, P_{4}, P_{6}\right]$} & $\frac{27 t^{4}+74 t^{2}+27}{36 t^{2}\left(t^{2}-1\right)^{2}}$ \\
& & {$[\pi / 3, \pi / 2, \pi / 3, \pi / 2]$} & $\frac{135 t^{2}-142 t+135}{144 t^{2}(t-1)^{2}}$ \\
$X_{6}^{(2)}$ & $t_{6}^{(2)}$ & {$\left[P_{3}, P_{4}, P_{6}\right]$} & $\frac{27 t^{2}-27 t+35}{36 t^{2}(t-1)^{2}}$ \\
& & {$[\pi / 4, \pi / 3, \pi / 4]$} & {$\left[P_{2}, P_{4}, P_{6}\right]$} \\
$X_{6}^{(3)}$ & $t_{6}^{(3)}$ & {$[\pi / 6, \pi / 6, \pi / 2]$} & $36 t^{2}\left(t^{2}-3 t+2\right)^{2}$ \\
$X_{6}^{(6)}$ & $t_{6}^{(6)}$ & {$\left[P_{0}, P_{4}, P_{7}, P_{6}\right]$} & $27 t^{4}-108$ \\
& & {$[\pi / 2, \pi / 3, \pi / 2, \pi / 2]$} & $135 t^{2}-103 t+108$ \\
$X_{6}^{+}$ & $t_{6}^{+}$ & {$\left[P_{0}, P_{4}, P_{6}\right]$} & $144 t^{2}(t-1)^{2}$ \\
\hline
\end{tabular}

6. Local uniformizing parameters. Our goal is to obtain explicit expansions of the uniformizing functions around the elliptic points and around some CM points. The purpose of this section is to make a first choice of local uniformizing parameters adapted to our functions (see definition below). 
Suppose that $P \in \mathcal{H}$ is any elliptic point of order $e$ for the $\bar{\Gamma}$-action. By definition, the isotropy group at $P, \bar{\Gamma}_{P}$, will be generated by a transformation $g \in \mathbf{P S L}(2, \mathbb{R})$ of order $e>1$. Let $G \in \Gamma \subseteq \mathbf{S L}(2, \mathbb{R})$ be a matrix defining $g$. Since in all our cases $-1 \in \Gamma$, we may take the matrix $G$ of order $2 e$, and since $g$ is an elliptic transformation, the matrix $G$ can be diagonalized. Let $H \in \mathbf{G L}(2, \mathbb{C})$ be such that $D:=H G H^{-1}=\left[\begin{array}{cc}\zeta & 0 \\ 0 & \zeta^{-1}\end{array}\right]$, where $\zeta$ is a $2 e$ th primitive root of unity. We denote by $h$ and $d$ the homographic transformations of $\mathbf{P}^{1}(\mathbb{C})$ defined by $H$ and $D$, respectively. Then

$$
h(g(z))=d(h(z))=\zeta^{2} h(z) .
$$

By evaluating $(*)$ at the points $z=P$ and $z=\bar{P}$, we obtain

$$
h(P)=h(g(P))=\zeta^{2} h(P), \quad h(\bar{P})=h(g(\bar{P}))=\zeta^{2} h(\bar{P}) .
$$

Since $e>1$, we have $\zeta^{2} \neq 1$, and since $h$ is a bijective mapping of $\mathbf{P}^{1}(\mathbb{C})$, we must have $h(P)=0$ and $h(\bar{P})=\infty($ or $h(P)=\infty$ and $h(\bar{P})=0)$. Hence,

$$
h(z)=k \frac{z-P}{z-\bar{P}} \quad\left(\text { or } h(z)=k \frac{z-\bar{P}}{z-P}\right)
$$

for some constant $k \in \mathbb{C}-\{0\}$ to be determined.

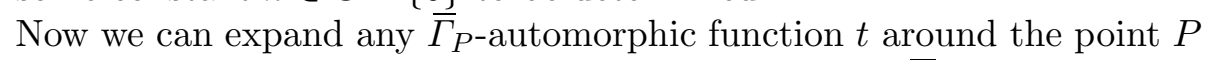
as a power series $T$ in the variable $h(z)=k(z-P) /(z-\bar{P})$ :

$$
t(z)=T(h(z))=\sum_{n=n_{0}}^{\infty} a_{n} h(z)^{n} .
$$

We shall have $T(h(z))=t(z)=t(g(z))=T(h(g(z)))=T\left(\zeta^{2} h(z)\right)$. Thus $a_{n}=0$ unless $n \equiv 0(\bmod e)$.

We extend these considerations to the case $e=1$ in the following definition.

Definition 6.1. A local parameter at a point $P \in \mathcal{H}$ for the $\bar{\Gamma}_{P \text {-action }}$ is any function

$$
q(z):=\left(k \frac{z-P}{z-\bar{P}}\right)^{e}
$$

where $e=\# \bar{\Gamma}_{P}$ is the order of the isotropy group at $P$ and $k \in \mathbb{C}-\{0\}$ is a constant. The local parameter $q$ is said to be adapted to a function $t=\sum_{n=m}^{\infty} a_{n e} q^{n}$ if, moreover, $a_{r e}=1$ in the case that $t-a_{0}$ has a zero of order $e r$ at $z=P$, or $a_{-r e}=1$ if $t$ has a pole of order $e r$ at $z=P$.

In order to obtain local parameters adapted to our functions, we review some classical facts regarding the Schwarzian functions. For this purpose, let us consider the hypergeometric function defined by the series

$$
F(a, b, c ; w)=\sum_{n=0}^{\infty} \frac{(a)_{n}(b)_{n}}{(c)_{n}} \frac{w^{n}}{n !}, \quad(a)_{n}:=a(a+1) \cdots(a+n-1)=\frac{\Gamma(a+n)}{\Gamma(a)},
$$

which converges for $|w|<1$ (cf. [7] and [11]). 
Theorem 6.2. Assume that $c \neq 1$. The functions $F(a, b, c ; w)$ and $w^{1-c} F(a-c+1, b-c+1,2-c ; w)$ are two linearly independent solutions of the hypergeometric differential equation

$$
w(1-w) D^{2}(f, w)+(c-(1+a+b) w) D(f, w)-a b f=0 .
$$

The function

$$
z=s(a, b, c ; w):=\frac{w^{1-c} F(a-c+1, b-c+1,2-c ; w)}{F(a, b, c ; w)}
$$

provides a conformal representation of the upper half $w$-plane $\mathcal{H}$ onto the interior of a triangle $[A, B, C]$ in the $z$-plane and establishes a homeomorphism between $\mathbb{R} \cup\{\infty\}$ and the boundary of the triangle. The vertices $A, B, C$ can be expressed in terms of Euler's gamma function as

$$
\begin{aligned}
& A=s(a, b, c ; 0)=0, \\
& B=s(a, b, c ; 1)=\frac{\Gamma(c-a) \Gamma(c-b) \Gamma(2-c)}{\Gamma(c) \Gamma(1-b) \Gamma(1-a)}, \\
& C=s(a, b, c ; \infty)=e^{\pi i(1-c)} \frac{\Gamma(c-a) \Gamma(b) \Gamma(2-c)}{\Gamma(c) \Gamma(b-c+1) \Gamma(1-a)} .
\end{aligned}
$$

The internal angles at these vertices are $\alpha \pi, \beta \pi, \gamma \pi$, where

$$
\alpha=1-c \neq 0, \quad \beta=c-a-b, \quad \gamma=b-a .
$$

In the next theorem we compare the triangle $[s(0), s(1), s(\infty)]$ with the triangles defining the functions $t_{6}^{+}, t_{6}^{(2)}$, and $t_{6}^{(3)}$. In each case, this will allow us to obtain the local constant $k$ of the adapted local parameter in closed form.

THEOREM 6.3. Let $t$ be one of the three uniformizing functions defined in Table 7 for which a fundamental half domain is a triangle; that is, $t_{6}^{+}$, $t_{6}^{(2)}$, and $t_{6}^{(3)}$. Let $\alpha \pi, \beta \pi, \gamma \pi$ be the internal angles at the vertices $A, B$, $C$ when $t$ takes the values $t(A)=0, t(B)=1, t(C)=\infty$, or $t(A)=1$, $t(B)=\infty, t(C)=0$, or $t(A)=\infty, t(B)=0, t(C)=1$. Then the local constants $k_{A}$ adapted to the functions $t$ are listed in Table 9.

Proof. First we explain the results for the case $t(A)=0$. By formal integration of the differential equation of the third order in Theorem 5.5, and taking into account that $t(A)=0$, it follows that there exists a normalized power series in two variables

$$
r(X, Y)=\sum_{n=1}^{\infty} a_{n e} X^{e n} Y^{e n}, \quad a_{e}=1,
$$

and a constant $\lambda \in \mathbb{C}$, such that

$$
t(z)=r\left(\lambda ; h_{1}(z)\right)=\sum_{n=1}^{\infty} a_{n e} \lambda^{e n} h_{1}^{e n}(z)
$$


Table 9. Local constants for the triangle functions

\begin{tabular}{ccccccc}
\hline$t$ & {$[A, B, C]$} & $e_{A}$ & $t(A)$ & $a, b, c$ & $\nu_{A}$ & $k_{A}$ \\
\hline$t_{6}^{+}$ & {$\left[P_{0}, P_{4}, P_{6}\right]$} & 2 & 0 & $\frac{1}{24}, \frac{7}{24}, \frac{1}{2}$ & $2^{3} \cdot 3^{2}$ & $i \frac{\sqrt{2}+\sqrt{3}}{2} \frac{\Gamma\left(\frac{7}{24}\right) \Gamma\left(\frac{11}{24}\right)}{\Gamma\left(\frac{19}{24}\right) \Gamma\left(\frac{23}{24}\right)}$ \\
$t_{6}^{+}$ & {$\left[P_{4}, P_{6}, P_{0}\right]$} & 6 & 1 & $\frac{1}{24}, \frac{13}{24}, \frac{5}{6}$ & $2^{-1} \cdot 3^{-2}$ & $\frac{2+\sqrt{3}-i}{12} \frac{\Gamma\left(\frac{1}{6}\right) \Gamma\left(\frac{7}{24}\right) \Gamma\left(\frac{19}{24}\right)}{\Gamma\left(\frac{5}{6}\right) \Gamma\left(\frac{11}{24}\right) \Gamma\left(\frac{23}{24}\right)}$ \\
$t_{6}^{+}$ & {$\left[P_{6}, P_{0}, P_{4}\right]$} & 4 & $\infty$ & $\frac{1}{24}, \frac{5}{24}, \frac{3}{4}$ & $2^{5} \cdot 3$ & $-\frac{(\sqrt{2}+\sqrt{3})(1+i)}{4 \sqrt{2}} \frac{\Gamma\left(\frac{1}{4}\right) \Gamma\left(\frac{13}{24}\right) \Gamma\left(\frac{17}{24}\right)}{\Gamma\left(\frac{3}{4}\right) \Gamma\left(\frac{19}{24}\right) \Gamma\left(\frac{23}{24}\right)}$ \\
$t_{6}^{(2)}$ & {$\left[P_{3}, P_{4}, P_{6}\right]$} & 4 & 0 & $\frac{1}{12}, \frac{1}{3}, \frac{3}{4}$ & $3^{2} \cdot 2^{-4}$ & $\frac{(1+\sqrt{3})(1+i)}{8} \frac{\Gamma\left(\frac{1}{4}\right) \Gamma\left(\frac{5}{12}\right)}{\Gamma\left(\frac{3}{4}\right) \Gamma\left(\frac{11}{12}\right)}$ \\
$t_{6}^{(2)}$ & {$\left[P_{4}, P_{6}, P_{3}\right]$} & 3 & 1 & $\frac{1}{12}, \frac{1}{3}, \frac{2}{3}$ & $2 \cdot 3^{-1}$ & $\frac{2+\sqrt{3}-i}{6} \frac{\Gamma\left(\frac{1}{3}\right)^{2} \Gamma\left(\frac{7}{12}\right)}{\Gamma\left(\frac{2}{3}\right)^{2} \Gamma\left(\frac{11}{12}\right)}$ \\
$t_{6}^{(2)}$ & {$\left[P_{6}, P_{3}, P_{4}\right]$} & 4 & $\infty$ & $\frac{1}{12}, \frac{5}{12}, \frac{3}{4}$ & $2^{8} \cdot 3$ & $-\frac{\sqrt{3}(1+i)}{4 \sqrt{2}} \frac{\Gamma\left(\frac{1}{3}\right) \Gamma\left(\frac{2}{3}\right) \Gamma\left(\frac{1}{4}\right)}{\Gamma\left(\frac{3}{4}\right) \Gamma\left(\frac{7}{12}\right) \Gamma\left(\frac{11}{12}\right)}$ \\
$t_{6}^{(3)}$ & {$\left[P_{2}, P_{4}, P_{6}\right]$} & 6 & 0 & $\frac{1}{12}, \frac{7}{12}, \frac{5}{6}$ & $2^{-3} \cdot 3^{-2}$ & $\frac{(1+\sqrt{3})(1+i)}{12} \frac{\Gamma\left(\frac{1}{6}\right) \Gamma\left(\frac{7}{12}\right)}{\Gamma\left(\frac{5}{6}\right) \Gamma\left(\frac{11}{12}\right)}$ \\
$t_{6}^{(3)}$ & {$\left[P_{4}, P_{6}, P_{2}\right]$} & 6 & 1 & $\frac{1}{12}, \frac{1}{4}, \frac{5}{6}$ & $2^{-3} \cdot 3^{-2}$ & $\frac{2+\sqrt{3}-i}{12} \frac{\Gamma\left(\frac{1}{6}\right) \Gamma\left(\frac{7}{12}\right)}{\Gamma\left(\frac{5}{6}\right) \Gamma\left(\frac{11}{12}\right)}$ \\
$t_{6}^{(3)}$ & {$\left[P_{6}, P_{2}, P_{4}\right]$} & 2 & $\infty$ & $\frac{1}{12}, \frac{1}{4}, \frac{1}{2}$ & 2 & $\frac{(1+\sqrt{3})(1-i)}{4} \frac{\Gamma\left(\frac{1}{4}\right) \Gamma\left(\frac{5}{12}\right)}{\Gamma\left(\frac{3}{4}\right) \Gamma\left(\frac{11}{12}\right)}$ \\
\hline
\end{tabular}

for any $z$ in a neighbourhood of $A$. Here we take $h_{1}(z):=(z-A) /(z-\bar{A})$. Observe that $\lambda$ is defined up to multiplication by an eth root of unity.

Consider the Schwarzian function $s(a, b, c ; w)$ determined by the angles $\alpha \pi, \beta \pi, \gamma \pi$, that is to say, $a=(1-\alpha-\beta-\gamma) / 2, b=(1-\alpha-\beta+\gamma) / 2$, $c=1-\alpha$. Since $r$ satisfies the conditions $r\left(\lambda ; h_{1}(A)\right)=0, r\left(\lambda ; h_{1}(B)\right)=1$, $r\left(\lambda ; h_{1}(C)\right)=\infty$, we can relate the inverse of the series defining $s(a, b, c ; w)$ to the series defining $t(z)$. A direct computation of the first terms in both series suffices to establish the following lemma.

Lemma 6.4. Let $u(a, b, c ; z)$ denote the inverse series of $s(a, b, c ; w)$. Then

$$
r\left(\zeta_{e} ; h_{1}(z)\right)=u\left(a, b, c ; h_{1}(z)\right)
$$

for any $z \in \mathbb{C}$ in the convergence domain and any eth root of unity $\zeta_{e}$.

To continue the calculation of $\lambda$, we may use either the condition $t(B)=1$ or, alternatively, the condition $t(C)=\infty$. In the first case, we obtain $1=$ $t(B)=r\left(\lambda ; h_{1}(B)\right)=r\left(1 ; \lambda h_{1}(B)\right)=r\left(\zeta_{e} ; \zeta_{e}^{-1} \lambda h_{1}(B)\right)$, and

$$
\zeta_{e}^{-1} \lambda h_{1}(B)=s(a, b, c ; 1)=\frac{\Gamma(c-a) \Gamma(c-b) \Gamma(2-c)}{\Gamma(c) \Gamma(1-b) \Gamma(1-a)} .
$$


We can conclude that

$$
\lambda=\zeta_{e} \frac{B-\bar{A}}{B-A} \frac{\Gamma(c-a) \Gamma(c-b) \Gamma(2-c)}{\Gamma(c) \Gamma(1-b) \Gamma(1-a)} .
$$

In the second case, we obtain

$$
\lambda=\zeta_{e} e^{\pi i(1-c)} \frac{C-\bar{A}}{C-A} \frac{\Gamma(c-a) \Gamma(b) \Gamma(2-c)}{\Gamma(c) \Gamma(b-c+1) \Gamma(1-a)} .
$$

Both values of $\lambda$ are equal and we may take $k_{A}$ to be the product of $\lambda$ by any eth root of unity. This is what we have done in Table 9 , where we have taken $\zeta_{e}=1$.

Now we explain the results for the cases $t(A)=1$ or $t(A)=\infty$. When $t(A)=1, t(B)=\infty, t(C)=0$, we change our function $t$ to $1-1 / t$; this function has the properties of the function $t$ in the preceding case, and we apply the same arguments. In the case $t(A)=\infty$, we change $t$ to $1 /(1-t)$ and proceed analogously.

Now, we compute local parameters for the two remaining uniformizing functions. To begin with, we state a result that relates the local constants for two points in $\mathcal{H}$ in the same $\bar{\Gamma}$-orbit.

Proposition 6.5. Let $P \in \mathcal{H}$ be a point of order $e \geq 1$ for the $\bar{\Gamma}$-action. For any $w=\left[\begin{array}{ll}a & b \\ c & d\end{array}\right] \in \Gamma \subseteq \mathbf{S L}(2, \mathbb{R})$, the local constants $k_{P}$ and $k_{w(P)}$ adapted to a $\bar{\Gamma}$-automorphic function $t$, at $P$ and $w(P)$, are related by

$$
k_{w(P)}^{e r}=k_{P}^{e r}\left(\frac{c P+d}{c \bar{P}+d}\right)^{e r},
$$

where the value of $r$ is given in Definition 6.1.

TheOREM 6.6. Table 10 lists the local constants $k_{P}$ adapted to the quadrilateral uniformizing functions $t_{6}$ and $t_{6}^{(6)}$ in neighbourhoods of the vertices $P$ of fundamental half domains and, also, in a neighbourhood of the SCM point $P_{0}$ for the function $t_{6}$.

Proof. We begin by explaining the computation for the point $P_{3}$ and the curve $X_{6}$. For this, we shall take into account the equation $t_{6}^{2}=t_{6}^{(2)}$ for the covering $X_{6} \rightarrow X_{6}^{(2)}$, which we computed in Theorem 4.3. First, we integrate the differential equations in Theorem 5.5 for the curves $X_{6}$ and $X_{6}^{(2)}$ in a neighbourhood of the point $P_{3}$ in the form

$$
\begin{aligned}
t_{6}^{(2)}(z) & =r_{6}^{(2)}\left(\lambda_{6}^{(2)} ; h_{1}(z)\right)=\sum_{n=1}^{\infty} a_{4 n}^{(2)} \lambda_{6}^{(2)^{4 n}} h_{1}^{4 n}(z), & & a_{4}^{(2)}=1, \\
t_{6}(z) & =r_{6}\left(\lambda_{6} ; h_{1}(z)\right)=\sum_{n=1}^{\infty} a_{2 n} \lambda_{6}^{2 n} h_{1}^{2 n}(z), & & a_{2}=1,
\end{aligned}
$$


Table 10. Local constants for the quadrilateral functions

\begin{tabular}{|c|c|c|c|c|c|}
\hline$t$ & $P$ & $e_{P}$ & $t(P)$ & $\nu_{P}$ & $k_{P}$ \\
\hline$t_{6}^{(6)}$ & $P_{0}$ & 2 & 0 & $2^{2} \cdot 3^{2}$ & $i \frac{\sqrt{2}+\sqrt{3}}{2 \sqrt{2}} \frac{\Gamma\left(\frac{7}{24}\right) \Gamma\left(\frac{11}{24}\right)}{\Gamma\left(\frac{19}{24}\right) \Gamma\left(\frac{23}{24}\right)}$ \\
\hline$t_{6}^{(6)}$ & $P_{4}$ & 3 & 1 & $3^{-1}$ & $\frac{2+\sqrt{3}+i}{12} \frac{\Gamma\left(\frac{1}{6}\right) \Gamma\left(\frac{7}{24}\right) \Gamma\left(\frac{19}{24}\right)}{\Gamma\left(\frac{5}{6}\right) \Gamma\left(\frac{11}{24}\right) \Gamma\left(\frac{23}{24}\right)}$ \\
\hline$t_{6}^{(6)}$ & $P_{7}$ & 2 & 2 & $2^{2} \cdot 3^{2}$ & $\frac{(2 \sqrt{3}+3 \sqrt{2})(1-\sqrt{2} i)}{12} \frac{\left.\Gamma\left(\frac{7}{24}\right) \Gamma\left(\frac{11}{24}\right)\right)}{\Gamma\left(\frac{19}{24}\right) \Gamma\left(\frac{23}{24}\right)}$ \\
\hline$t_{6}^{(6)}$ & $P_{6}$ & 2 & $\infty$ & $2^{2}$ & $i \frac{\sqrt{2}+\sqrt{3}}{4} \frac{\Gamma\left(\frac{1}{4}\right) \Gamma\left(\frac{13}{24}\right) \Gamma\left(\frac{17}{24}\right)}{\Gamma\left(\frac{3}{4}\right) \Gamma\left(\frac{19}{24}\right) \Gamma\left(\frac{23}{24}\right)}$ \\
\hline$t_{6}$ & $P_{0}$ & 1 & $i$ & $2^{2} \cdot 3$ & $i \frac{\sqrt{2}+\sqrt{3}}{2} \frac{\Gamma\left(\frac{7}{24}\right) \Gamma\left(\frac{11}{24}\right)}{\Gamma\left(\frac{19}{24}\right) \Gamma\left(\frac{23}{24}\right)}$ \\
\hline$t_{6}$ & $P_{2}$ & 3 & -1 & $3^{-1}$ & $\frac{1+(2+\sqrt{3}) i}{6 \sqrt[3]{2}} \frac{\Gamma\left(\frac{1}{3}\right)^{2} \Gamma\left(\frac{7}{12}\right)}{\Gamma\left(\frac{2}{3}\right)^{2} \Gamma\left(\frac{11}{12}\right)}$ \\
\hline$t_{6}$ & $P_{3}$ & 2 & 0 & $3 \cdot 2^{-2}$ & $\frac{(1+\sqrt{3})(1+i)}{8} \frac{\Gamma\left(\frac{1}{4}\right) \Gamma\left(\frac{5}{12}\right)}{\Gamma\left(\frac{3}{4}\right) \Gamma\left(\frac{11}{12}\right)}$ \\
\hline$t_{6}$ & $P_{4}$ & 3 & 1 & $3^{-1}$ & $\frac{2+\sqrt{3}-i}{6 \sqrt[3]{2}} \frac{\Gamma\left(\frac{1}{3}\right)^{2} \Gamma\left(\frac{7}{12}\right)}{\Gamma\left(\frac{2}{3}\right)^{2} \Gamma\left(\frac{11}{12}\right)}$ \\
\hline$t_{6}$ & $P_{6}$ & 2 & $\infty$ & $2^{4}$ & $\frac{\sqrt{3}(1-i)}{4 \sqrt{2}} \frac{\Gamma\left(\frac{1}{3}\right) \Gamma\left(\frac{2}{3}\right) \Gamma\left(\frac{1}{4}\right)}{\Gamma\left(\frac{3}{4}\right) \Gamma\left(\frac{7}{12}\right) \Gamma\left(\frac{11}{12}\right)}$ \\
\hline
\end{tabular}

where $e_{6}^{(2)}=4, e_{6}=2$ are the orders of the isotropy groups at $P_{3}$, and $h_{1}(z)=\left(z-P_{3}\right) /\left(z-\bar{P}_{3}\right)$.

Now, we impose the condition that these series expansions satisfy the equation $t_{6}^{2}=t_{6}^{(2)}$; this gives us the relation $\lambda_{6}^{4}=\lambda_{6}^{(2)^{4}}$, and so a local constant $\lambda_{6}$ adapted to $t_{6}$ at $P_{3}$ is, up to multiplication by a fourth root of unity, the local constant $\lambda_{6}^{(2)}$ adapted to $t_{6}^{(2)}$ at $P_{3}$.

To determine this fourth root of unity up to sign we note that the value of $t_{6}$ at $P_{0}$ may be obtained by estimating the series at the point $z=P_{0}$, because the series is absolutely convergent at this point. But, if for $\lambda_{6}$ we take $\pm i$ times the value of $\lambda_{6}^{(2)}$ listed in Table 9 , then the value of the series at $P_{0}$ approximates $-i$ rather than $i$, as it should do. Thus, we must take \pm 1 times the value of $\lambda_{6}^{(2)}$, as done in Table 10 .

The local parameters adapted to $t_{6}$ at the points $P_{4}, P_{6}$ and $P_{0}$, and the ones adapted to $t_{6}^{(6)}$ at $P_{0}, P_{4}$, and $P_{6}$, are computed similarly. The relationship between the ones we want to compute and the ones we use to compute them are calculated taking into account the equations given in Theorem 4.3, and they are as follows:

$$
\begin{aligned}
& 2 \lambda_{6}^{(6)}\left(P_{0}\right)^{2}=\lambda_{6}^{+}\left(P_{0}\right)^{2}, \quad \lambda_{6}^{(6)}\left(P_{4}\right)^{6}=-\lambda_{6}^{+}\left(P_{4}\right)^{6}, \quad \lambda_{6}^{(6)}\left(P_{6}\right)^{4}=-\lambda_{6}^{+}\left(P_{6}\right)^{4} \text {, } \\
& \lambda_{6}\left(P_{0}\right)^{2}=2 \lambda_{6}^{(6)}\left(P_{0}\right)^{2}, \quad 2 \lambda_{6}\left(P_{4}\right)^{3}=\lambda_{6}^{(2)}\left(P_{4}\right)^{3}, \quad \lambda_{6}\left(P_{6}\right)^{4}=\lambda_{6}^{(2)}\left(P_{6}\right)^{4} .
\end{aligned}
$$


To determine the roots of unity that we need, we note that although we may estimate $t_{6}$ at $P_{0}$, because the series expansions of $t_{6}$ are absolutely convergent at this point, we cannot estimate $t_{6}^{(6)}$ at $P_{4}$ nor at $P_{6}$ with the development of $t_{6}^{(6)}$ at $P_{0}$, because this series is not absolutely convergent at any of these points. But we may estimate the value of $t_{6}^{(6)}$ along the segment joining $P_{0}$ to $P_{6}$, where $t_{6}^{(6)}$ must take negative real values, because in the open segment $\left[P_{0}, P_{6}\right)$ the development of $t_{6}^{(6)}$ at $P_{0}$ is absolutely convergent. This adjusts the root of unity for the local constant $\lambda_{6}\left(P_{0}\right)$.

For the other, we proceed in a similar manner; but we need to mention that, in the integration of the differential equation for $t_{6}$ in a neighbourhood of the SCM point $P_{0}$, the solution depends on two parameters and not on only one as in the other cases. The relation between them is obtained from the equation that relates $t_{6}$ to $t_{6}^{+}$or, alternatively, the equation that relates $t_{6}$ to $t_{6}^{(6)}$.

Finally, it remains to determine the local parameters adapted to $t_{6}$ at $P_{2}$ and adapted to $t_{6}^{(6)}$ at $P_{7}$. For this, we shall use Proposition 6.5. Note that

$$
w_{2}\left(P_{0}\right)=P_{7} \quad \text { and } \quad w_{6}\left(P_{4}\right)=P_{2} ;
$$

thus, we may relate the local parameters adapted to $t_{6}^{+}$at $P_{0}$ and $P_{7}$ to obtain the latter, and then lift it to a local parameter adapted to $t_{6}^{(6)}$ at $P_{7}$; and, similarly, relating the local parameters adapted to $t_{6}^{(6)}$ at $P_{4}$ and $P_{2}$, we obtain the latter, and then we lift it to a local parameter adapted to $t_{6}$ at $P_{2}$.

At this point, it would be natural to consider the adapted local parameter

$$
q_{A}(z)=\left(k_{A} \frac{z-A}{z-\bar{A}}\right)^{e_{A}}
$$

as a uniformizing variable in the neighbourhood of the point $A$. By doing this, we would obtain series developments

$$
\begin{aligned}
& t(z)=\sum_{n=1}^{\infty} b_{n} q^{n}, \quad b_{n}:=a_{n e}, b_{1}=1, \quad \text { if } t(A)=0, \\
& t(z)=t(A)+\sum_{n=1}^{\infty} b_{n} q^{n}, \quad b_{n}:=a_{n e}, b_{1}=1, \quad \text { if } t(A) \neq 0, \infty, \\
& t(z)=\sum_{n=-1}^{\infty} b_{n} q^{n}, \quad b_{n}:=a_{n e}, b_{-1}=1, \quad \text { if } t(A)=\infty .
\end{aligned}
$$

However, the arithmetic properties of the coefficients $b_{n}$ can be improved. With this aim in mind, the functions $q_{A}$ will be slightly modified in Section 7 . 
7. Local expansions. Series expansions for the uniformizing functions considered in the preceding sections will be given in a neighbourhood of the vertices of the fundamental half domain; all of them are elliptic points. Moreover, the uniformizing function $t_{6}$ will be developed in a neighbourhood of the SCM point $P_{0}$. Observe that $P_{0}$ is an elliptic point for $X_{6}^{(6)}$ and $X_{6}^{+}$, but it is not elliptic for $X_{6}, X_{6}^{(2)}$ and $X_{6}^{(3)}$.

CASE $t(P) \neq \infty$. We begin by studying the uniformizing functions $t$ at the points $P$ where they take a finite value $v$. First, we consider developments of the shape

$$
t(z)=\sum_{n=0}^{\infty} b_{n}^{\prime} \frac{q(z)^{n}}{(e n) !}, \quad b_{1}^{\prime}=e !
$$

Next we renormalize the function $q$. We replace $q$ by $\nu^{-1} q$, where the values of $\nu$ are listed in Tables 9 and 10. Thus,

$$
t(z)=\sum_{n=0}^{\infty} b_{n}^{\prime \prime} \frac{q(z)^{n}}{(e n) !}, \quad b_{1}^{\prime \prime}=\nu e !
$$

Finally, we define the factor $\mathfrak{n}_{v}=\nu e$ !, where $v=t(P)$, and normalize the generating function $t$ by $t\left(P, q_{P} ; z\right):=\mathfrak{n}_{v}^{-1} t(z)$ so that

$$
t\left(P, q_{P} ; z\right)=\sum_{n=0}^{\infty} c_{n} \frac{q_{P}(z)^{n}}{(e n) !}, \quad c_{1}=1, \quad q_{P}(z)=\frac{1}{\nu_{P}}\left(k_{P} \frac{z-P}{z-\bar{P}}\right)^{e_{P}}
$$

where the values of $e_{P}, k_{P}$ and $\nu_{P}$ are listed in Tables 9 and 10 .

CASE $t(P)=\infty$. Finally, we study the uniformizing functions $t$ at the point $P_{6}$, where all of them take the value $\infty$. First, we consider developments of the shape

$$
t(z)=\sum_{n=-1}^{\infty} b_{n}^{\prime} \frac{q(z)^{n}}{(2 e(n+2)) !}, \quad b_{-1}^{\prime}=(2 e) !
$$

Next we renormalize the function $q$. We replace $q$ by $\nu^{-1} q$, where the values of $\nu$ are listed in Tables 9 and 10. Thus,

$$
t(z)=\sum_{n=-1}^{\infty} b_{n}^{\prime \prime} \frac{q(z)^{n}}{(2 e(n+2)) !}, \quad b_{-1}^{\prime \prime}=\nu(2 e) !
$$

Finally, we define the factor $\mathfrak{n}_{\infty}=\nu(2 e)$ ! and normalize the generating function $t$ by $t\left(P, q_{P} ; z\right):=\mathfrak{n}_{\infty}^{-1} t(z)$ so that

$t\left(P, q_{P} ; z\right)=\sum_{n=-1}^{\infty} c_{n} \frac{q_{P}(z)^{n}}{(2 e(n+2)) !}, \quad c_{-1}=1, \quad q_{P}(z)=\frac{1}{\nu_{P}}\left(k_{P} \frac{z-P}{z-\bar{P}}\right)^{e_{P}}$,

where the values of $e_{P}, k_{P}$ and $\nu_{P}$ are listed in Tables 9 and 10 . 
The following lemma, whose proof is left to the reader, justifies the choice of the local functions $q_{P}(z)^{n} /(2 e(n+2))$ !.

Lemma 7.1. Let

$$
f(q):=\sum_{n=1}^{\infty} \frac{a_{n}}{(e n) !} q^{n}
$$

be a power series such that $a_{1}=e$ ! and $a_{n} \in \mathbb{Z}$. Define

$$
\frac{1}{f(q)}=\sum_{n=-1}^{\infty} \frac{b_{n}}{(2 e(n+2)) !} q^{n} .
$$

Then $b_{n} \in(2 e) ! \mathbb{Z}$ for any $n \geq-1$.

We note that each generating function $t\left(P, q_{P} ; z\right)$ is a representative of the homothety class of the corresponding function $t$. The representatives depend on the point $P$ and the relations between them are compiled in Table 11.

Table 11. Local uniformizing functions $t\left(P, q_{P} ; z\right)=\mathfrak{n}_{t(P)}^{-1} \cdot t(z)$

\begin{tabular}{cccccc}
\hline$X$ & $X_{6}^{+}$ & $X_{6}^{(2)}$ & $X_{6}^{(3)}$ & $X_{6}^{(6)}$ & $X_{6}$ \\
$t$ & $t_{6}^{+}$ & $t_{6}^{(2)}$ & $t_{6}^{(3)}$ & $t_{6}^{(6)}$ & $t_{6}$ \\
\hline $\mathfrak{n}_{\infty}$ & $3870720=$ & $30965760=$ & $48=2^{4} \cdot 3$ & $96=2^{5} \cdot 3$ & $384=2^{7} \cdot 3$ \\
& $2^{12} \cdot 3^{3} \cdot 5 \cdot 7$ & $2^{15} \cdot 3^{3} \cdot 5 \cdot 7$ & & & \\
& $t_{6}^{+}\left(P_{6}, q_{P_{6}}\right)$ & $t_{6}^{(2)}\left(P_{6}, q_{P_{6}}\right)$ & $t_{6}^{(3)}\left(P_{6}, q_{P_{6}}\right)$ & $t_{6}^{(6)}\left(P_{6}, q_{P_{6}}\right)$ & $t_{6}\left(P_{6}, q_{P_{6}}\right)$ \\
\hline $\mathfrak{n}_{0}$ & $144=2^{4} \cdot 3^{2}$ & $\frac{27}{2}=2^{-1} \cdot 3^{3}$ & $10=2 \cdot 5$ & $72=2^{3} \cdot 3^{2}$ & $\frac{3}{2}=2^{-1} \cdot 3$ \\
& $t_{6}^{+}\left(P_{0}, q_{P_{0}}\right)$ & $t_{6}^{(2)}\left(P_{3}, q_{P_{3}}\right)$ & $t_{6}^{(3)}\left(P_{2}, q_{P_{2}}\right)$ & $t_{6}^{(6)}\left(P_{0}, q_{P_{0}}\right)$ & $t_{6}\left(P_{3}, q_{P_{3}}\right)$ \\
\hline $\mathfrak{n}_{1}$ & $40=2^{3} \cdot 5$ & $4=2^{2}$ & $10=2 \cdot 5$ & 2 & 2 \\
& $t_{6}^{+}\left(P_{4}, q_{P_{4}}\right)$ & $t_{6}^{(2)}\left(P_{4}, q_{P_{4}}\right)$ & $t_{6}^{(3)}\left(P_{4}, q_{P_{4}}\right)$ & $t_{6}^{(6)}\left(P_{4}, q_{P_{4}}\right)$ & $t_{6}\left(P_{4}, q_{P_{4}}\right)$ \\
\hline $\mathfrak{n}_{2}$ & $*$ & $*$ & $*$ & $72=2^{3} \cdot 3^{2}$ & $*$ \\
& $*$ & $*$ & $*$ & $t_{6}^{(6)}\left(P_{7}, q_{P_{7}}\right)$ & $*$ \\
\hline $\mathfrak{n}_{-1}$ & $*$ & $*$ & $*$ & $*$ & 2 \\
& $*$ & $*$ & $*$ & $*$ & $t_{6}\left(P_{2}, q_{P_{2}}\right)$ \\
\hline $\mathfrak{n}_{i}$ & $*$ & $*$ & $*$ & $*$ & $12=2^{2} \cdot 3$ \\
& $*$ & $*$ & $*$ & & $t_{6}\left(P_{0}, q_{P_{0}}\right)$ \\
\hline & & $*$ & $*$ & $*$
\end{tabular}

Since they might be useful for further studies (as in the case of the modular $j$-function), we provide the starting coefficients for some developments of the complex uniformizing function $t_{6}$ of the curve $X_{6}$. 
The coefficients $c_{n}(1 \leq n \leq 10)$ of $t_{6}\left(P_{3}, q_{P_{3}} ; z\right)$ :

$$
\begin{aligned}
1 & =1 \\
0 & =0 \\
-48 & =-2^{4} \cdot 3 \\
0 & =0 \\
27504 & =2^{4} \cdot 3^{2} \cdot 191 \\
0 & =0 \\
-64498392 & =-2^{3} \cdot 3^{2} \cdot 7 \cdot 127973 \\
0 & =0 \\
436272183216 & =2^{4} \cdot 3^{4} \cdot 23 \cdot 229 \cdot 63913 \\
0 & =0
\end{aligned}
$$

The coefficients $c_{n}(0 \leq n \leq 10)$ of $t_{6}\left(P_{4}, q_{P_{4}} ; z\right)$ :

$$
\begin{aligned}
1 / 2 & =2^{-1} \\
1 & =1 \\
20 & =2^{2} \cdot 5 \\
1356 & =2^{2} \cdot 3 \cdot 113 \\
227040 & =2^{5} \cdot 3 \cdot 5 \cdot 11 \cdot 43 \\
74611380 & =2^{2} \cdot 3 \cdot 5 \cdot 1243523 \\
42574294080 & =2^{6} \cdot 3^{2} \cdot 5 \cdot 17 \cdot 19 \cdot 45767 \\
38683567274400 & =2^{5} \cdot 3^{2} \cdot 5^{2} \cdot 5372717677 \\
52554612744944640 & =2^{10} \cdot 3^{2} \cdot 5 \cdot 11 \cdot 23 \cdot 4507937111 \\
101782604056899960000 & =2^{6} \cdot 3^{4} \cdot 5^{4} \cdot 139 \cdot 226002762361 \\
270629344957362042528000 & =2^{8} \cdot 3^{4} \cdot 5^{3} \cdot 29 \cdot 16126171 \cdot 223259851
\end{aligned}
$$

The coefficients $c_{n}(0 \leq n \leq 10)$ of $t_{6}\left(P_{0}, q_{P_{0}} ; z\right)$ :

$$
\begin{aligned}
i / 12 & =-i \cdot(1+i)^{-4} \cdot 3^{-1} \\
1 & =1 \\
-12 i & =i \cdot(1+i)^{4} \cdot 3 \\
-226 & =-2 \cdot 113 \\
5664 i & =(1+i)^{10} \cdot 3 \cdot 59 \\
160728 & =2^{3} \cdot 3 \cdot 37 \cdot 181 \\
-5467296 i & =-(1+i)^{10} \cdot 3 \cdot 56951 \\
-211472208 & =-2^{4} \cdot 3^{5} \cdot 109 \cdot 499 \\
9193300992 i & =-i \cdot(1+i)^{20} \cdot 3^{2} \cdot 571 \cdot 1747 \\
445513958784 & =2^{7} \cdot 3^{3} \cdot 128910289 \\
-23734590202368 i & =-(1+i)^{18} \cdot 3^{4} \cdot 15919 \cdot 35951
\end{aligned}
$$


The coefficients $c_{n}(-1 \leq n \leq 10)$ of $t_{6}\left(P_{6}, q_{P_{6}} ; z\right)$ :

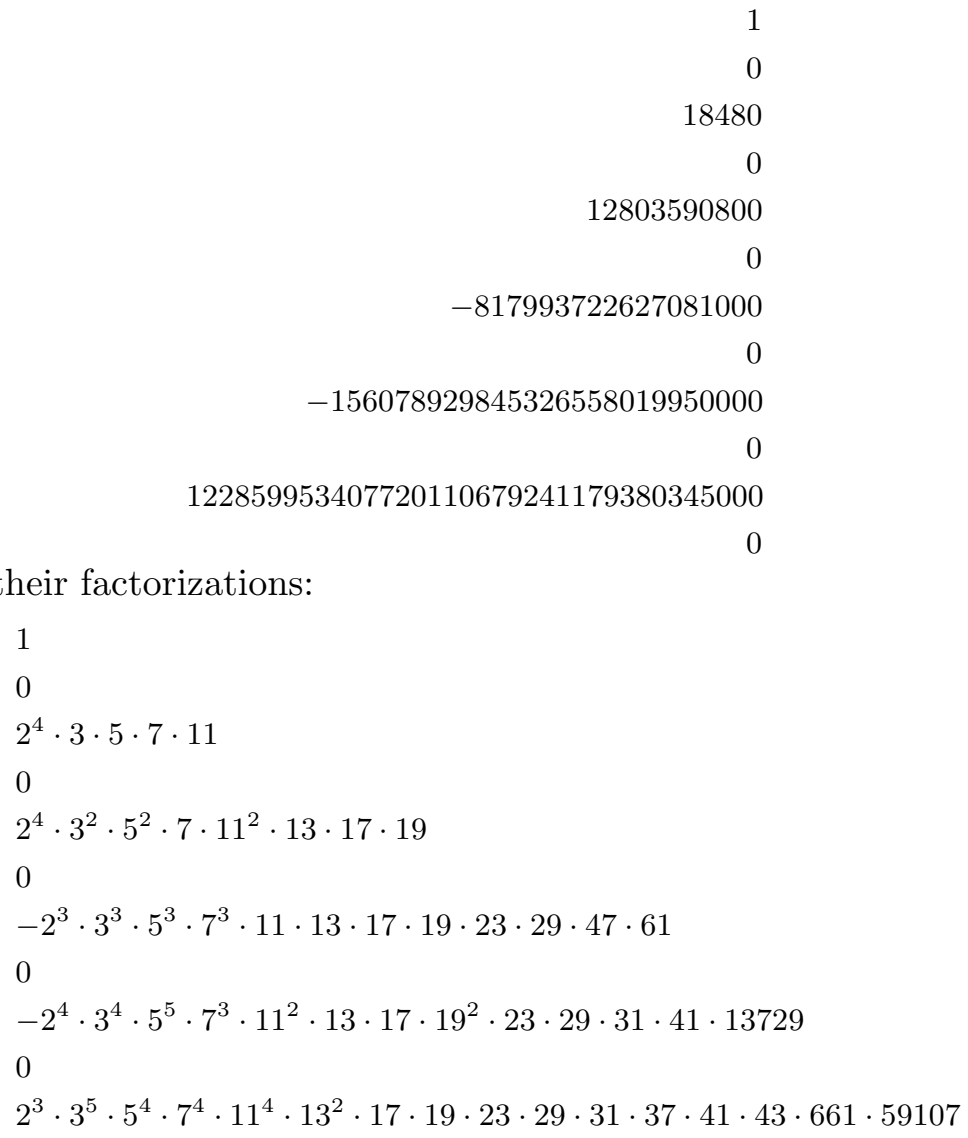

and their factorizations:

In [3], the preceding method has been applied in the modular case, providing natural series expansions for all the triangle modular functions (including the elliptic modular function $j$ ) around any elliptic point or cusp. Thus, in this case, each of these functions also supplies a family of natural arithmetic developments.

8. Canonical models. In this section we derive explicit canonical models for the curves $X_{6}, X_{6}^{(2)}, X_{6}^{(3)}, X_{6}^{(6)}, X_{6}^{+}$. Actually, the models we have computed up to now are complex models or, more precisely, models defined over the field $\mathbb{Q}\left(\zeta_{12}\right)$, where $\zeta_{12}$ is a 12 th primitive root of unity (cf. Corollary 8.2 and Remark 8.3).

Note that $X_{6}$ has no real points; in particular, although it is of genus zero and defined over $\mathbb{Q}$, the field $\mathbb{Q}\left(X_{6}\right)$ cannot be generated by a single function valued in $\mathbf{P}^{1}(\mathbb{C})$. 
We know by Shimura [10] that there exist canonical rational models $j_{6}: \bar{\Gamma}_{6} \backslash \mathcal{H} \rightarrow X_{6}(\mathbb{C}), j_{6}^{(d)}: \bar{\Gamma}_{6}^{(d)} \backslash \mathcal{H} \rightarrow X_{6}^{(d)}(\mathbb{C}), d=2,3,6$, and $j_{6}^{+}: \bar{\Gamma}_{6}^{+} \backslash \mathcal{H} \rightarrow$ $X_{6}^{+}(\mathbb{C})$, which are compatible with the projections on the left hand side and the covering mappings on the right hand side.

Lemma 8.1. The points $j_{6}\left(P_{2}\right)$ and $j_{6}\left(P_{4}\right)$ are rational over $\mathbb{Q}(\sqrt{-3})$. The points $j_{6}\left(P_{3}\right)$ and $j_{6}\left(P_{6}\right)$ are rational over $\mathbb{Q}(\sqrt{-1})$. The points $j_{6}\left(P_{0}\right)$ and $j_{6}\left(P_{7}\right)$ are rational over $\mathbb{Q}(\sqrt{-6}, \sqrt{-3})$.

Proof. The points $j_{6}\left(P_{2}\right)$ and $j_{6}\left(P_{4}\right)$ are elliptic of order 3 ; therefore, they are rational over the field $\mathbb{Q}(\sqrt{-3})$ (cf. [4]). Analogously, the points $j_{6}\left(P_{3}\right)$ and $j_{6}\left(P_{6}\right)$ are elliptic of order 2 ; therefore, they are rational over the field $\mathbb{Q}(\sqrt{-1})$. On the other hand, the points $j_{6}\left(P_{0}\right)$ and $j_{6}\left(P_{7}\right)$ are complex multiplication points for the quadratic field $\mathbb{Q}(\sqrt{-6})$. They are the fixed points for the embeddings $\mathbb{Q}(\sqrt{-6}) \hookrightarrow \mathbb{H}_{6}$ given, respectively, by $\sqrt{-6} \mapsto-3 \mathrm{~J}+\mathrm{K}$ and $\sqrt{-6} \mapsto \mathrm{I}-3 \mathrm{~J}$. According to the model of Shimura, we have

$$
\begin{aligned}
\mathbb{Q}\left(j_{6}\left(P_{0}\right)\right) \cdot \mathbb{Q}(\sqrt{-6}) & =\mathbb{Q}\left(j_{6}\left(P_{7}\right)\right) \cdot \mathbb{Q}(\sqrt{-6}) \\
& =\operatorname{HCF}(\mathbb{Q}(\sqrt{-6}))=\mathbb{Q}(\sqrt{-6}, \sqrt{-3}) .
\end{aligned}
$$

COROLlary 8.2. The models given by the functions $t_{6}^{+}, t_{6}^{(2)}, t_{6}^{(3)}, t_{6}^{(6)}$, and $t_{6}$ are defined over $k:=\mathbb{Q}\left(\zeta_{24}\right)=\mathbb{Q}(\sqrt{-3}, \sqrt{-1}, \sqrt{2})$.

Proof. Since all of our curves are of genus zero and all of them have $k$-rational points, their fields of rational functions over $k$ are generated by a single function. Now, since the automorphism group of the field is $\operatorname{PSL}(2, k)$, the generator is uniquely determined by its value at three $k$-rational points. But our functions $t_{6}^{+}, t_{6}^{(2)}, t_{6}^{(3)}, t_{6}^{(6)}$ and $t_{6}$ take $k$-rational values $0,1, \infty$ at points that are $k$-rational in the canonical model. Therefore, they generate the function fields over $k$.

REMARK 8.3. Actually, the minimal field of definition of the points $j_{6}\left(P_{0}\right)$ and $j_{6}\left(P_{7}\right)$ is $\mathbb{Q}(\sqrt{-3})$ (cf. Theorem 8.7). For the minimal field of definition of each of the preceding models see the discussion preceding Theorem 8.7.

Now, we prove that the model computed for $X_{6}^{+}$is the canonical model.

Proposition 8.4. For the curve $X_{6}^{+}$we have:

(a) $j_{6}^{+}\left(P_{0}\right)=j_{6}^{+}\left(P_{7}\right), j_{6}^{+}\left(P_{2}\right)=j_{6}^{+}\left(P_{4}\right), j_{6}^{+}\left(P_{3}\right)=j_{6}^{+}\left(P_{6}\right) \in X_{6}^{+}(\mathbb{Q})$.

(b) $X_{6}^{+}(\mathbb{Q}) \simeq \mathbf{P}^{1}(\mathbb{Q})$.

(c) $\mathbb{Q}\left(X_{6}^{+}\right)=\mathbb{Q}\left(t_{6}^{+}\right)$.

Proof. The points $j_{6}^{+}\left(P_{0}\right)=j_{6}^{+}\left(P_{7}\right), j_{6}^{+}\left(P_{2}\right)=j_{6}^{+}\left(P_{4}\right)$ and $j_{6}^{+}\left(P_{3}\right)=$ $j_{6}^{+}\left(P_{6}\right)$ are the only points of $X_{6}^{+}$that are elliptic, and they are of different orders; therefore, they must be rational. Since $X_{6}^{+}$is defined over $\mathbb{Q}$ and of 
genus 0 , we obtain (b). Finally, $t_{6}^{+}$takes rational values at the three rational points $j_{6}^{+}\left(P_{0}\right), j_{6}^{+}\left(P_{4}\right), j_{6}^{+}\left(P_{6}\right)$ and we obtain $(\mathrm{c})$.

Next, we show that there are $\mathbb{Q}$-rational points in any of the curves $X_{6}^{(2)}$, $X_{6}^{(3)}$ and $X_{6}^{(6)}$.

Proposition 8.5. For the curves $X_{6}^{(2)}, X_{6}^{(3)}$ and $X_{6}^{(6)}$, we have:

(a) $j_{6}^{(2)}\left(P_{2}\right)=j_{6}^{(2)}\left(P_{4}\right), j_{6}^{(2)}\left(P_{0}\right)=j_{6}^{(2)}\left(P_{7}\right) \in X_{6}^{(2)}(\mathbb{Q})$.

(b) $j_{6}^{(3)}\left(P_{3}\right)=j_{6}^{(3)}\left(P_{6}\right), j_{6}^{(3)}\left(P_{0}\right)=j_{6}^{(3)}\left(P_{7}\right) \in X_{6}^{(3)}(\mathbb{Q})$.

(c) $j_{6}^{(6)}\left(P_{2}\right)=j_{6}^{(6)}\left(P_{4}\right), j_{6}^{(6)}\left(P_{3}\right)=j_{6}^{(6)}\left(P_{6}\right) \in X_{6}^{(6)}(\mathbb{Q})$.

Therefore, there exist isomorphisms $X_{6}^{(2)}(\mathbb{Q}) \simeq \mathbf{P}^{1}(\mathbb{Q}), X_{6}^{(3)}(\mathbb{Q}) \simeq \mathbf{P}^{1}(\mathbb{Q})$ and $X_{6}^{(6)}(\mathbb{Q}) \simeq \mathbf{P}^{1}(\mathbb{Q})$.

Proof. (a) The point $j_{6}^{(2)}\left(P_{2}\right)=j_{6}^{(2)}\left(P_{4}\right)$ is the unique elliptic point of order 3 of $X_{6}^{(2)}$; therefore, it must be rational. Moreover, the cover $X_{6}^{(2)} \rightarrow$ $X_{6}^{+}$ramifies only at the points $j_{6}^{+}\left(P_{0}\right)$ and $j_{6}^{+}\left(P_{4}\right)$, which are rational. Since one of the ramification points, namely $j_{6}^{(2)}\left(P_{4}\right)$, is rational, the other must also be rational; that is, $j_{6}^{(2)}\left(P_{0}\right)=j_{6}^{(2)}\left(P_{7}\right) \in X_{6}^{(2)}(\mathbb{Q})$.

(b) We argue similarly, with $P_{4}$ replaced by $P_{6}$.

(c) Now, $j_{6}^{(6)}\left(P_{2}\right)=j_{6}^{(6)}\left(P_{4}\right)$ is the unique elliptic point of order 3 , and the ramification points are $j_{6}^{+}\left(P_{4}\right)$ and $j_{6}^{+}\left(P_{6}\right)$.

COROLlary 8.6. There exist automorphic functions $u_{2}, u_{3}, u_{6}$ such that $\mathbb{Q}\left(X_{6}^{(2)}\right)=\mathbb{Q}\left(u_{2}\right), \mathbb{Q}\left(X_{6}^{(3)}\right)=\mathbb{Q}\left(u_{3}\right)$ and $\mathbb{Q}\left(X_{6}^{(6)}\right)=\mathbb{Q}\left(u_{6}\right)$.

To compute these functions $u_{2}, u_{3}, u_{6}$, we shall take in account the ramification of the coverings. Since $X_{6}^{(2)} \rightarrow X_{6}^{+}$is ramified only at the points such that $t_{6}^{+}$takes the values 0,1 , the field extension $\mathbb{Q}\left(X_{6}^{(2)}\right) \mid \mathbb{Q}\left(X_{6}^{+}\right)=$ $\mathbb{Q}\left(t_{6}^{+}\right)$is ramified exactly at $t_{6}^{+}$and $t_{6}^{+}-1$. Therefore, we can choose $u_{2}$ such that $u_{2}^{2}=r_{2} t_{6}^{+}\left(t_{6}^{+}-1\right)^{-1}$, where $r_{2}$ is a square free integer. On the other hand, since $X_{6}^{(3)} \rightarrow X_{6}^{+}$is ramified only at the points such that $t_{6}^{+}$takes the values $0, \infty$, the field extension $\mathbb{Q}\left(X_{6}^{(3)}\right) \mid \mathbb{Q}\left(X_{6}^{+}\right)$is ramified exactly at $t_{6}^{+}$and $\infty$. Therefore, we can choose $u_{3}$ such that $u_{3}^{2}=r_{3} t_{6}^{+}$, where $r_{3}$ is a square free integer. And finally, since $X_{6}^{(6)} \rightarrow X_{6}^{+}$is ramified only at the points such that $t_{6}^{+}$takes the values $1, \infty$, the field extension $\mathbb{Q}\left(X_{6}^{(6)}\right) \mid \mathbb{Q}\left(X_{6}^{+}\right)$is ramified exactly at $t_{6}^{+}-1$ and $\infty$. Therefore, we can choose $u_{6}$ such that $u_{6}^{2}=r_{6}\left(t_{6}^{+}-1\right)$, where $r_{6}$ is a square free integer. Moreover, the three quadratic subfields of the field $\mathbb{Q}\left(X_{6}^{(3)}\right) \cdot \mathbb{Q}\left(X_{6}^{(6)}\right)=\mathbb{Q}\left(X_{6}\right)$ being $\mathbb{Q}\left(X_{6}^{(2)}\right)$, $\mathbb{Q}\left(X_{6}^{(3)}\right)$ and $\mathbb{Q}\left(X_{6}^{(6)}\right)$, we may take the constants $r_{2}, r_{3}$ and $r_{6}$ such that one of them equals the product of the other two. 
Now, observe that the equations in Theorem 4.3 imply the following ones:

$$
\left(\frac{t_{6}^{(2)}+1}{t_{6}^{(2)}-1}\right)^{2}=\frac{t_{6}^{+}}{t_{6}^{+}-1}, \quad\left(2 t_{6}^{(3)}-1\right)^{2}=t_{6}^{+}, \quad\left(t_{6}^{(6)}-1\right)^{2}=1-t_{6}^{+} .
$$

Therefore, we have

$$
u_{2}=\sqrt{r_{2}} \frac{t_{6}^{(2)}+1}{t_{6}^{(2)}-1}, \quad u_{3}=\sqrt{r_{3}}\left(2 t_{6}^{(3)}-1\right), \quad u_{6}=\sqrt{-r_{6}}\left(t_{6}^{(6)}-1\right) .
$$

Next we compute the constants $r_{2}, r_{3}$ and $r_{6}$. Since $t_{6}^{(2)}$ takes values in $\mathbb{Q}(\sqrt{-1})$ for three $\mathbb{Q}(\sqrt{-1})$-rational points of $X_{6}^{(2)}$, namely, the points $j_{6}^{(2)}\left(P_{3}\right), j_{6}^{(2)}\left(P_{4}\right), j_{6}^{(2)}\left(P_{6}\right)$, we have $\mathbb{Q}(\sqrt{-1})\left(X_{6}^{(2)}\right)=\mathbb{Q}(\sqrt{-1})\left(t_{6}^{(2)}\right)$. Therefore,

$$
\mathbb{Q}(\sqrt{-1})\left(u_{2}\right)=\mathbb{Q}(\sqrt{-1})\left(X_{6}^{(2)}\right)=\mathbb{Q}(\sqrt{-1})\left(t_{6}^{(2)}\right)=\mathbb{Q}(\sqrt{-1})\left(\sqrt{r_{2}} u_{2}\right) .
$$

Thus, $\sqrt{r_{2}} \in \mathbb{Q}(\sqrt{-1})$. Working similarly for $t_{6}^{(3)}$ and $X_{6}^{(3)}$, we find that $\sqrt{r_{3}} \in \mathbb{Q}(\sqrt{-3})$. Since $r_{2}$ and $r_{3}$ are square free, we must have $r_{2}=-1$ or $r_{2}=1$, and $r_{3}=-3$ or $r_{3}=1$. To discard the value $r_{3}=1$, we observe that $\mathbb{Q}\left(X_{6}\right)=\mathbb{Q}\left(u_{3}, u_{6}\right)$, and if $r_{3}=1$, then the two functions $u_{3}, u_{6}$ would take rational values $(1,0)$ at $P_{4}$, while $j_{6}\left(P_{4}\right)$ is not a rational point of $X_{6}$. This fixes $r_{3}=-3$. Similarly, if $r_{2}=1$, then the two functions $u_{2}$ and $1 / u_{6}$ would take rational values $(1,0)$ at $P_{6}$, while $j_{6}\left(P_{6}\right)$ is not a rational point of $X_{6}$ and $\mathbb{Q}\left(X_{6}\right)=\mathbb{Q}\left(u_{2}, 1 / u_{6}\right)$. This fixes $r_{2}=-1$ and $r_{6}=3$.

We are now in a position to make precise the minimal field of definition of the models in Corollary 8.2: it is $\mathbb{Q}$ for $t_{6}^{+} ; \mathbb{Q}(\sqrt{-1})$ for $t_{6}^{(2)} ; \mathbb{Q}(\sqrt{-3})$ for $t_{6}^{(3)}$ and $t_{6}^{(6)}$; and $\mathbb{Q}(\sqrt{-3}, \sqrt{-1})$ for $t_{6}$.

We have proven the following result. In particular, our functions satisfy the algebraic equation for $X_{6}$ given by Ihara (cf. [6]).

TheOREM 8.7. The functions $j_{6}^{+}, j_{6}^{(2)}, j_{6}^{(3)}, j_{6}^{(6)}: \mathcal{H} \rightarrow \mathbf{P}^{1}(\mathbb{C})$ given by

$$
\begin{gathered}
j_{6}^{+}=t_{6}^{+}, \quad j_{6}^{(2)}=u_{2}=\sqrt{-1} \frac{t_{6}^{(2)}+1}{t_{6}^{(2)}-1}, \\
j_{6}^{(3)}=u_{3}=\sqrt{-3}\left(2 t_{6}^{(3)}-1\right), \quad j_{6}^{(6)}=u_{6}=\sqrt{-3}\left(t_{6}^{(6)}-1\right),
\end{gathered}
$$

and the function $j_{6}: \mathcal{H} \rightarrow \mathbf{P}^{2}(\mathbb{C})$ given by

$$
j_{6}=\left(u_{3}: u_{6}: 1\right)=\left(u_{2}: 1: 1 / u_{6}\right)=\left(1: 1 / u_{2}: 1 / u_{3}\right)
$$

define canonical models of the curves $X_{6}^{+}, X_{6}^{(2)}, X_{6}^{(3)}, X_{6}^{(6)}$, and $X_{6}$, respectively. For the canonical model of the curve $X_{6}$, we have $\mathbb{Q}\left(X_{6}\right)=\mathbb{Q}\left(j_{6}\right)=$ 
$\mathbb{Q}\left(u_{3}, u_{6}\right)=\mathbb{Q}\left(u_{2}, u_{6}\right)=\mathbb{Q}\left(u_{2}, u_{3}\right)$ and the equations

$$
u_{3}^{2}+u_{6}^{2}+3=0, \quad u_{2}^{2}+1+\frac{3}{u_{6}^{2}}=0, \quad 1+\frac{1}{u_{2}^{2}}+\frac{3}{u_{3}^{2}}=0
$$

provide a set of affine rational charts for the projective curve $X_{6} / \mathbb{Q}$. Moreover, $j_{6}\left(P_{0}\right)=(0:-\sqrt{-3}: 1)$ and $j_{6}\left(P_{7}\right)=(0: \sqrt{-3}: 1)$.

\section{References}

[1] M. Alsina and P. Bayer, Quaternion Orders, Quadratic Forms and Shimura Curves, CRM Monogr. Ser. 22, Amer. Math. Soc., 2004.

[2] P. Bayer, Uniformization of certain Shimura curves, in: Differential Galois Theory, T. Crespo and Z. Hajto (eds.), Banach Center Publ. 58, Inst. Math., Polish Acad. Sci., 2002, 13-26.

[3] P. Bayer and A. Travesa, Uniformization of triangle modular curves, Publ. Mat., to appear.

[4] N. D. Elkies, Shimura curves computations, in: Lecture Notes in Comput. Sci. 1423, Springer, 1998, 1-49.

[5] A. R. Forsyth, Theory of Differential Equations, Vol. III, Cambridge Univ. Press, 1902.

[6] A. Kurihara, On some examples of equations defining Shimura curves and the Mumford uniformization, J. Fac. Sci. Univ. Tokyo 25 (1979), 277-301.

[7] Z. Nehari, Conformal Mapping, Dover, 1952.

[8] A. P. Ogg, Real Points on Shimura Curves, Progr. Math. 35, Birkhäuser, 1983, 277-307.

[9] G. Sansone and J. Gerretsen, Lectures on the Theory of Functions of a Complex Variable. II Geometric Theory, Wolters-Noordhoff, Groningen, 1969.

[10] G. Shimura, Construction of class fields and zeta functions of algebraic curves, Ann. of Math. 85 (1967), 58-159.

[11] M. Yoshida, Fuchsian Differential Equations, Max-Planck-Institut für Mathematik and Vieweg, 1987.

Facultat de Matemàtiques

Universitat de Barcelona

Gran Via de les Corts Catalanes 585

E-08007, Barcelona, Spain

E-mail: bayer@ub.edu

travesa@ub.edu

Received on 12.7.2005

and in revised form on 8.9.2006 